\title{
Component- and System-Level Degradation Modeling of Digital Instrumentation and Control Systems Based on a Multi-State Physics
}

Modeling Approach

\author{
Wei Wang ${ }^{1}$, Francesco Di Maio ${ }^{1}$, Enrico Zio ${ }^{1,2}$ \\ ${ }^{I}$ Energy Department, Politecnico di Milano, Via La Masa 34, 20156 Milano, Italy \\ ${ }^{2}$ Chair on System Science and the Energetic Challenge, Fondation EDF-Electricite de France, Ecole \\ Centrale Paris and Supelec, Chatenay-Malabry Cedex, 92295 Paris, France
}

\begin{abstract}
A system-level degradation modeling is proposed for the reliability assessment of digital Instrumentation and Control (I\&C) systems in Nuclear Power Plants (NPPs). At the component level, we focus on the reliability assessment of a Resistance Temperature Detector (RTD), which is an important digital I\&C component used to guarantee the safe operation of NPPs. A Multi-State Physics Model (MSPM) is built to describe this component degradation progression towards failure and Monte Carlo (MC) simulation is used to estimate the probability of sojourn in any of the previously defined degradation states, by accounting for both stochastic and deterministic processes that affect the degradation progression.
\end{abstract}

The MC simulation relies on an integrated modeling of stochastic processes with deterministic aging of components that results to be fundamental for estimating the joint cumulative probability distribution of finding the component in any of the possible degradation states.

The results of the application of the proposed degradation model to a digital I\&C system of literature are compared with the results obtained by a Markov Chain Model (MCM). The integrated stochastic-deterministic process here proposed to drive the MC simulation is viable to integrate component-level models into a system-level model that would consider inter-system or/and inter-component dependencies and uncertainties. Keywords: Multi-State Physics Modeling; Digital I\&C System; System-Level Model; Component-Level Model; Degradation State Probability. 


\section{ABBREVIATIONS}

PSA

NPP

I\&C

MSPM

$\mathrm{MCM}$

$\mathrm{MC}$

RTD

RPS

BPL

LCL

PTS

ESS

RTB

$\mathrm{CDF}$

PDF

\section{NOTATIONS}

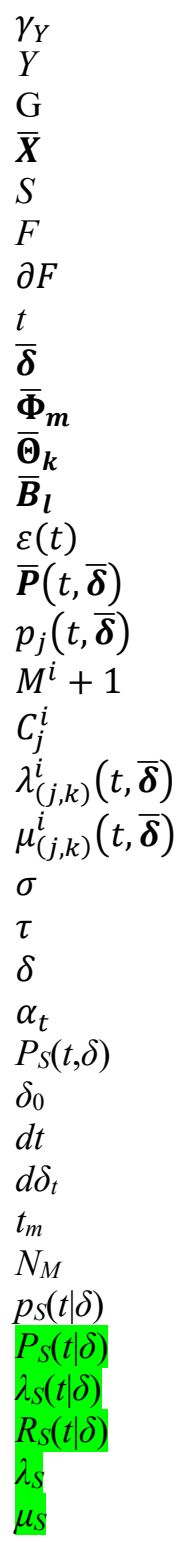

Probabilistic Safety Analysis

Nuclear Power Plant

Instrumentation and Control

Multi-State Physics Model(ing)

Markov Chain Model

Monte Carlo

Resistance Temperature Detector

Reactor Protection System

Bistable Processor Logic

Local Coincidence Logic

Partial Tripping Signal

Emergency Shutdown Signal

Reactor Trip Breaker

Cumulative Distribution Function

Probability Density Function

Failure threshold

Physical variable

Limit-state function

Vector of system parameters

Safety domain

Failure domain

Failure boundary

Time

Vector of physical parameters

Vector of $m$-dimensional manufacturing features

Vector of $k$-dimensional stochastic parameters

Vector of $l$-dimensional external parameters

Error term

State probability vector obtained from MSPM

State probability of state $j$ in MSPM

Number of states in $i$-th component/module-level MSPM

Degradation state $j$ of component (module) $i$

Failure rate of component (module) $i$ from state $C_{j}^{i}$ to $C_{k}^{i}$

Repair rate of component (module) $i$ from state $C_{k}^{i}$ to $C_{j}^{i}$

RTD measurement accuracy

RTD response time

RTD air gap size

Scale factor

CDF of the RTD New-to-drift failure mode

Initial air gap size

Time interval

Noise of air gap size

Mission time

Simulation times

Conditional PDF given air gap size interval

Conditional CDF given air gap size interval

Conditional failure rate given air gap size interval

Conditional reliability of RTD given air gap size interval

Failure rate in RTD-MCM

Repair rate in RTD-MCM 


$\begin{array}{ll}P_{S}(t) & \text { Unreliability obtained from RTD-MCM } \\ N+1 & \text { Number of layers in system-level MSPM } \\ L^{l} & \text { Layer } l \text { in system-level MSPM } \\ M^{l}+1 & \text { Number of degradation states of layer } l \\ L_{m}^{l} & \text { Degradation state } m \text { of layer } l \\ \mu_{L_{m}^{l} \rightarrow L^{0}}(t, \overline{\boldsymbol{\delta}}) & \text { Repair rate from state } L_{m}^{l} \text { to state } L^{0} \\ \lambda_{L_{m}^{l} \rightarrow L_{n}^{\omega}}(t, \overline{\boldsymbol{\delta}}) & \text { Failure rate from state } L_{m}^{l} \text { to state } L_{n}^{\omega} \\ \lambda_{L_{m}^{l} \rightarrow L^{N}}(t, \overline{\boldsymbol{\delta}}) & \text { Failure rate from state } L_{m}^{l} \text { to system failure state } L^{N} \\ \lambda_{\mathrm{B}} & \text { BPL failure rate } \\ \lambda_{\mathrm{L}} & \text { LCL failure rate } \\ \beta & \text { Common cause factor } \\ \lambda_{\mathrm{BC}} & \text { BPL common cause failure rate } \\ \lambda_{\mathrm{LC}} & \text { LCL common cause failure rate } \\ \lambda_{\mathrm{R}} & \text { RTB failure rate } \\ P(t \mid \delta) & \text { RPS unreliability obtained from RPS-MSPM } \\ P(t) & \text { RPS unreliability obtained from RPS-MCM }\end{array}$

\section{Introduction}

In support to the implementation of risk-informed decision-making approaches, Probabilistic Safety Analysis (PSA) of modernizing Nuclear Power Plants (NPPs) demands for detailed dynamic models of digital Instrumentation and Control (I\&C) systems that can adequately represent digital components failure modes and quantify their contribution to the overall risk of the NPPs (Aldemir et al., 2007; Aldemir et al., 2006).

To this aim, dynamic methods are being increasingly integrated into existing PSA frameworks for digital I\&C systems reliability assessment, such as: Dynamic Flowgraph Methodology (DFM) (Guarro et al., 2012; Aldemir et al., 2006; Aldemir et al., 2009), Markov/cell-to-cell mapping technique (CCMT) (Aldemir et al., 2006; Aldemir et al., 2009; Zhou et al., 2014), Petri Net (Lee et al., 2006; Kim and Kim, 2014), Bayesian Networks (Boudali and Dugan, 2006; Broy et al., 2011), Dynamic Fault Tree (DFT) (Dehlinger and Dugan, 2008), Dynamic Event Tree (DET) (Bucci et al., 2006) and Fuzzy CMeans (FCM) clustering method (Di Maio et al., 2011; Zio and Di Maio, 2009). On a system level, these methods can be used to tackle the twofold purpose of PSA: on one side, the identification of the system failure domain and, on the other side,

the quantification of the system failure probability. 
With respect to the latter, given a failure threshold $\gamma_{Y}$ not to be exceeded by a safety-relevant physical variable $Y$ during the system operation, a limit-state function $G$ can be defined as:

$$
G=G\left(\bar{X}, \gamma_{Y}\right)=Y(\bar{X})-\gamma_{Y}
$$

where $\bar{X}=\left\{X_{1}, X_{2}, \cdots, X_{n}\right\}$ defines the system parameters and operational conditions. This leads to the definition of a system safety domain $S=\left\{\bar{X}: G\left(\bar{X}, \gamma_{Y}\right)<0\right\}$ and of a system failure domain $F=\left\{\bar{X}: G\left(\bar{X}, \gamma_{Y}\right)>0\right\}$, that are partitioned by a system failure boundary $\partial F=G\left(\bar{X}, \gamma_{Y}\right)=0$, for a given $\gamma_{Y}$.

The identification of the failure domain $F$ is crucial especially when the system dynamics is complex and its component reliability assessment cannot be described by a Boolean, discrete and abrupt physics of failure, but rather by a multi-valued, and continuous degradation model as it is for digital I\&C systems (Li et al., 2012; Lin et al., 2015; Lisnianski and Levitin, 2003). The biggest challenge to be overcome for devising realistic and effective degradation models consists in the collection of component reliability data that are, often, affected by multiple and competing failure modes that are difficult to be untangled and reduced to a single-lumped failure criterion analysis that would leverage the degradation modeling task. To avoid simplification and overlooking of failure interdependencies, we propose to resort to a Multi-State Physics Modeling (MSPM) approach at the component level, which can be easily upscaled for system-level degradation modeling. The MSPM approach is based on the structure of Markov (or semi-Markov) modeling for the quantification of components reliability measures (Unwin et al., 2011; Unwin et al., 2012; Rocco and Zio, 2013; Fleming et al., 2010). Recently, the MSPM approach has been proposed for modeling nuclear component degradation by accounting for both the effects of stochastic parameters affecting the degradation and the environmental parameters with their uncertainties (Lin et al., 2015; Di Maio et al., 2015).

In this study, a component-level MSPM model for a digital I\&C system is developed by integrating in the model both the stochastic and the deterministic processes that affect component degradation. The physical variable $Y$ to be considered for the failure domain $F$ identification is given in Eq. (2) (Kaiser and Gebraeel, 2009): 


$$
Y=Y(\bar{X})=f(t, \overline{\boldsymbol{\delta}})+\varepsilon(t)=f\left(t, \overline{\boldsymbol{\Phi}}_{m}, \overline{\boldsymbol{\Theta}}_{k}, \overline{\boldsymbol{B}}_{l}\right)+\varepsilon(t)
$$

where $t$ is the deterministic aging time, $\bar{\delta}$ is a collection of physical parameters affecting the degradation process that can be seen as composed by $\overline{\boldsymbol{\Phi}}_{m}=\left\{\varphi_{1}, \cdots, \varphi_{m}\right\}$ which is a vector of $m$-dimensional manufacturing features that affect the degradation (e.g., burn-in, contamination, etc.), $\overline{\boldsymbol{\Theta}}_{k}=\left\{\theta_{1}, \cdots, \theta_{k}\right\}$ which is a vector of $k$-dimensional stochastic parameters that account for the components variability (e.g., nominal frequency stability, calibration error after maintenance, etc.), $\overline{\boldsymbol{B}}_{l}=\left\{\beta_{1}, \cdots, \beta_{l}\right\}$ which is a vector of $l$-dimensional external parameters that capture the variability of timevarying operating and environmental conditions (e.g., temperature, flux, etc.), and $\varepsilon(t)$ that is an error term that captures noise and disturbances. In principle, a component response surface to any possible different setting of degradation features (stochastic and external parameters, and error terms) can be built (with infinite computational resources) such that the safety domain $S$ can be partitioned from the failure domain $F$ by setting a failure threshold $\gamma_{Y}$.

In this work, a Monte Carlo (MC) simulation is used to estimate the transition probabilities among the degradation states of MSPM and drive, by random walks, the stochastic process of the evolution of the air gap size in time and the deterministic evolution of the component aging on the response surface for the identification of the limit surface of the drift event of a Resistance Temperature Detector (RTD) that is embedded into a digital I\&C system of a NPP.

Finally, as for traditional PSA (where system-level models are developed by combining or replacing subsystem or component models in the overall structure of a Fault Tree (FT) or an Event Tree (ET) (Aldemir et al., 2007; Aldemir et al., 2009; Gulati and Dugan, 1997)), the system failure probability of the digital I\&C system is quantified by upscaling the component-level MSPM into a system-level model that considers the inter-system or/and inter-component dependencies and the aleatory or/and epistemic uncertainties affecting each component behavior.

The rest of paper is as follows. Section 2 presents the illustration of componentlevel MSPM for a digital I\&C component (e.g., sensor, reactor trip breaker, CPU, etc.) 
and the comparison of the results obtained by the Markov Chain method. Section 3 presents the system-level MSPM framework of a digital I\&C system in a NPP. In section 4 conclusions are drawn.

\section{The Component-Level MSPMs}

In this study, the digital I\&C System of reference, whose reliability assessment is required for the failure domain identification and the related risk quantification of a NPP, is a typical NPP Reactor Protection System (RPS). Its objective is to trigger reactor emergency shutdown as soon as an anomaly is detected in the measurements of some relevant signals (i.e., primary coolant pressure, temperature, etc.). As shown in Fig. 1, this digital I\&C system is composed of two redundant channels (A and B). Each channel consists of one signal sensor (S-A and S-B), one Bistable Processor Logic (BPL) subsystem (BPL-A and BPL-B), and one Local Coincidence Logic (LCL) subsystem (LCL-A and LCL-B).

An independent signal sensor and an independent BPL is installed in each channel. If any of the two redundant measured signals exceeds a safety threshold value, a Partial Tripping Signal (PTS) is measured from the corresponding BPL, e.g. a PTS from BPL$\mathrm{A}$ is measured because $\mathrm{S}-\mathrm{A}$ exceeds the tripping value. The signal processing proceeds only if both channels produce the PTS: each PTS from a BPL is sent to both LCL-A and LCL-B, which process information by an "AND" gate. In other words, an Emergency Shutdown Signal (ESS) is produced only when receiving two PTSs from different BPLs; ESSs, then, activates the Reactor Trip Breaker (RTB), when at least one ESS is triggered, i.e., the information is processed by an "OR" gate. Once the RTB is activated, the power supply system and Control Rod Drive Mechanism (CRDM) which are connected with the RTB come into use to control the power of the reactor. 


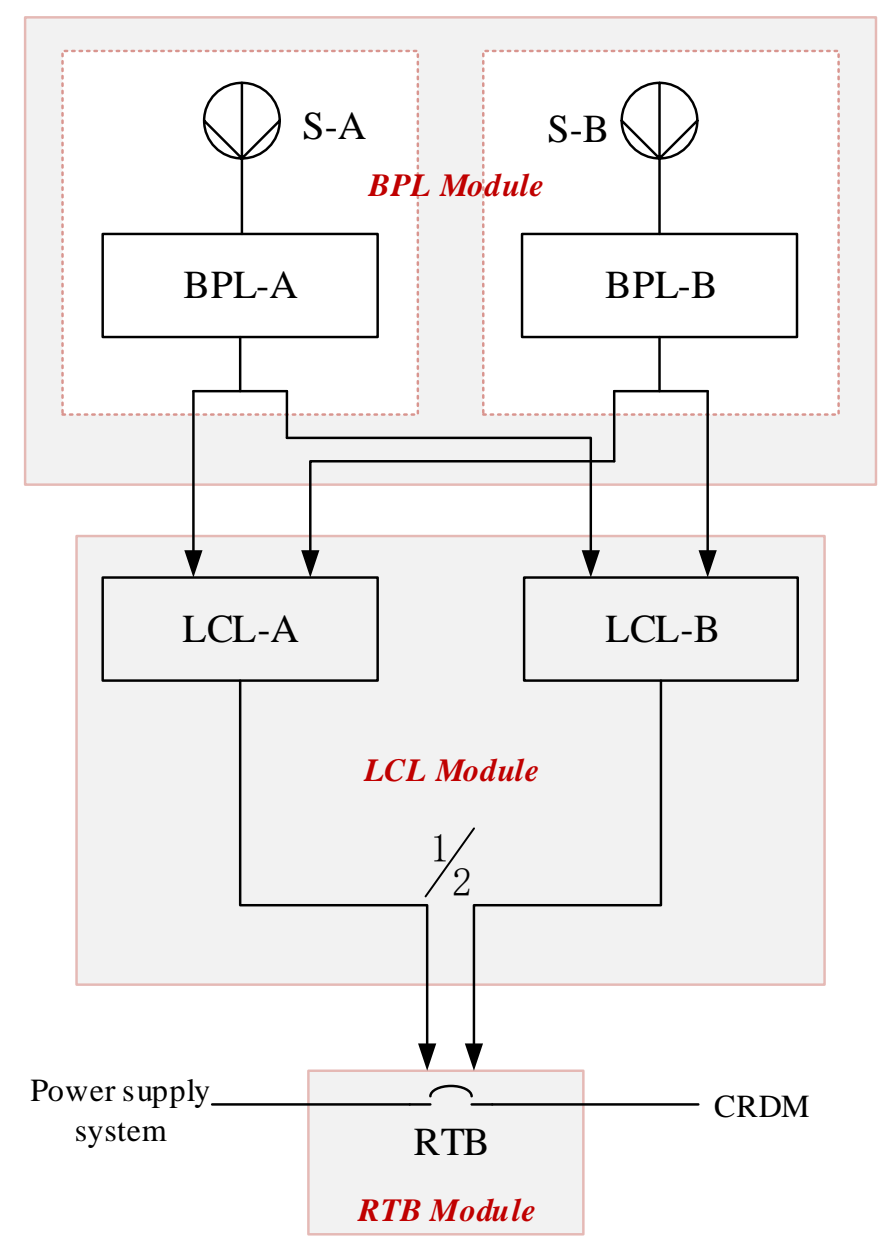

Fig. 1 A typical RPS (Wang et al., 2015)

The system can be decomposed into modules to reduce the complexity of systemlevel modeling based on the functions the embedded components are devised for and the failure effects they produce on the system. According to the RPS scheme of Fig. 1, the RPS modules are identified:

- The BPL Module, consists of two groups of components: sensor and BPL (i.e., "S-A and BPL-A" and "S-B and BPL-B"); these components are connected in series and their failure effects on the system can be combined.

- The LCL Module consists of the two LCLs (i.e., LCL-A and LCL-B). Since the ESS is triggered only when both LCLs simultaneously receive two PTSs from the two BPLs, this module is highly dependent of the BPL module.

- The RTB Module.

Without loss of generality, let us consider and build the component-level MSPM of the module "S-A and BPL-A". It is worth mentioning that, for a comprehensive and 
exhaustive analysis, a component-level MSPM of each module shown in Fig. 1 should be built with great detail and embedded into the system-level MSPM, as we shall see in Section 3.

Each component-level model is a MSPM where the dynamics of component degradation is represented by transitions among a finite number $M$ of degradation states at any time instant $t$ and any value of the affecting parameters $\bar{\delta}$ (Li et al., 2012; Di Maio et al., 2015); similarly to a Markov Chain Model (MCM), a state probability $p$ is assigned to each degradation state, that is collectively represented by a state probability vector $\overline{\boldsymbol{P}}(t, \overline{\boldsymbol{\delta}})=\left\{p_{0}(t, \overline{\boldsymbol{\delta}}), p_{1}(t, \overline{\boldsymbol{\delta}}), \cdots p_{j}(t, \overline{\boldsymbol{\delta}}), \cdots, p_{M}(t, \overline{\boldsymbol{\delta}})\right\}$ for all the $M$ states.

- In general terms, we model each $i$-th component with a graph with $M^{i}+1$ nodes, each of which identifies a state of the component degradation progression. Herein, " $C_{0}^{i}$ " and " $C_{M}^{i}$ " represent the "New" and "Failed" states of the $i$-th component in the system, whereas any other state " $C_{j}^{i}$ ", $j=1,2, \cdots, M^{i}$ is an intermediate degradation state, where the component is partially functioning.

- State " $C_{0}^{i}$ " is selected as the initial state at time $t=0$, i.e., the component is "New" at $t=0$.

- $\lambda_{(j, k)}^{i}(t, \overline{\boldsymbol{\delta}}), j=1,2, \cdots, M^{i}, k=1,2, \cdots, M^{i}$, and $j \neq k$ is the transition rate of the $i$-th component degradation model from state " $C_{j}^{i}$ " to state " $C_{k}^{i}$ ", with respect to the time and the degradation affecting factor vector $\overline{\boldsymbol{\delta}}$.

- Components can be repaired even if not "Failed", i.e., repair rate $\mu_{(j, k)}^{i}(t, \overline{\boldsymbol{\delta}})$, $j=1,2, \cdots, M^{i}, k=1,2, \cdots, M^{i}$, and $j \neq k$ can be foreseen between the intermediate transition states " $C_{j}^{i}$ " and " $C_{k}^{i}$ ".

An example of MSPM of a generic single component $i$ is sketched in Fig. 2, below.

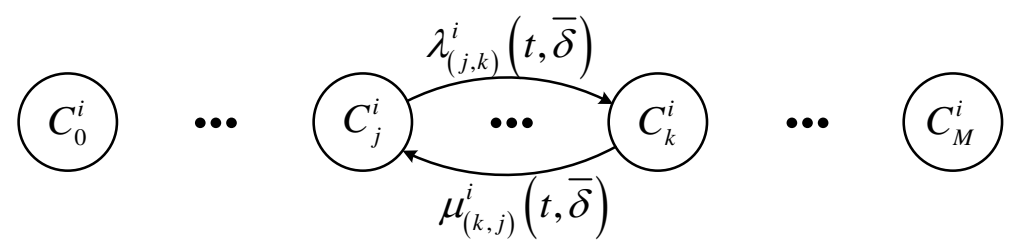

Fig. 2 MSPM degradation model of single component $i$ 


\subsection{The Sensor MSPM}

Different types of sensors are employed for measuring temperatures, pressures, levels and flows in NPP safety systems, such as Resistance Temperature Detectors (RTDs) (Montalvo et al., 2014; Hashemian, 2011; Yun et al., 2012), pressure transmitters (Hashemian, 2011), eddy current sensors (García-Martín et al., 2011; Uchanin and Najda, 2011) and optical fiber sensors (Ferdinand et al., 2013). Without loss of generality, we assume that the S-A and S-B of the digital I\&C system of Fig. 1 are RTDs. We focus on RTDs because they are critical components, whose effectiveness in promptly detecting anomalous temperature changes greatly support plant operators in the monitoring of NPP operational conditions and guide counteracting measure to avoid system failure. This is why RTDs must properly generate accurate and timely data (Baraldi et al., 2015). The NPP plant power level is, indeed, set based on the information gathered from RTDs: the better the performance of these process instrumentations in terms of measurement accuracy, the larger the power rate with enough margin from the system failure domain $F$ (hence, the better the plant economics) (Yun et al., 2012).

To build the RTD-MSPM model (as sketched in Fig. 3), the sensor failure modes have to be identified (e.g., bias (Uren et al., 2015), drift (Uren et al., 2015; Garvey and Hines, 2006), performance degradation (Fernandeza et al., 2015), freezing (Boskovic and Mehra, 2002), and calibration error (Castello et al., 2014)) and assumptions are made for the subsequent quantitative analysis:

- Six degradation states, besides the sensor functioning state $\left(C_{0}^{R T D}\right)$, are set and organized into RTD-MSPM model: $C_{1}^{R T D}$ corresponds to Bias, $C_{2}^{R T D}$ corresponds to Drift, $C_{3}^{R T D}$ corresponds to (performance) Degradation, $C_{4}^{R T D}$ corresponds to Freezing, $C_{5}^{R T D}$ corresponds to Calibration Error and $C_{6}^{R T D}$ corresponds to Complete Failure. Transitions can occur between state $C_{0}^{R T D}$ and any of the other degradation states, with transition rate $\lambda_{(0, j)}^{R T D}(t, \bar{\delta})$ (here $j$ 
$=1,2,3,4,5,6)$ and between degradation states with transition rate $\lambda_{(j, k)}^{R T D}(t, \bar{\delta})$ (here $j, k=1,2,3,4,5,6$ and $\left.j \neq k\right)$. It is assumed that repair is just carried out from each degradation state to state $C_{0}^{R T D}$, with repair rate $\mu_{(j, 0)}^{R T D}(t, \bar{\delta})$

- The initial state at time $t=0$ is sensor functioning state $C_{0}^{R T D}$.

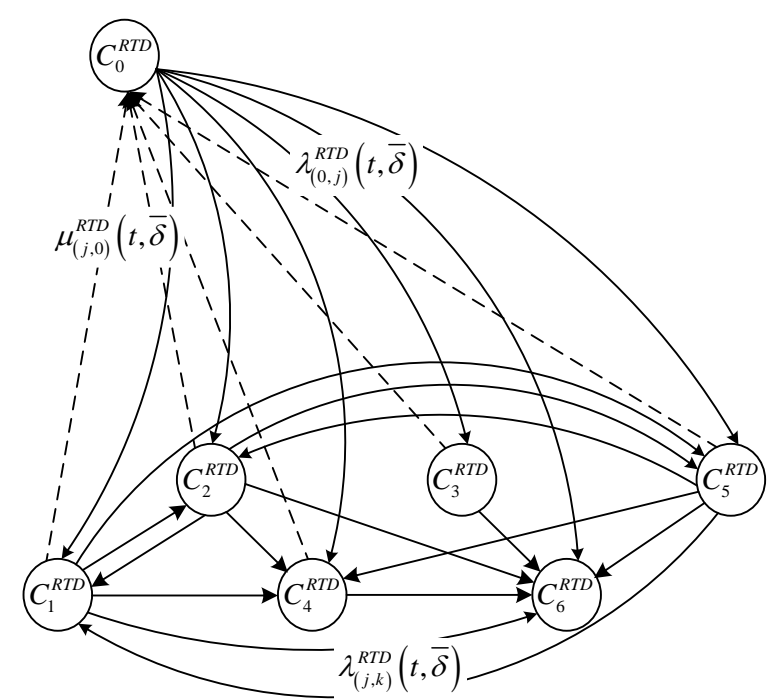

Fig. 3 Schematic diagram of the RTD-MSPM

The RTD-MSPM is further simplified as in Fig. 4, where $C_{0}^{R T D}$ is the RTD functioning state and $C_{2}^{R T D}$ is the RTD failure degradation state due to drift with failure rate $\lambda_{(0,2)}^{R T D}(t, \bar{\delta})$ and repair rate $\mu_{(2,0)}^{R T D}(t, \bar{\delta})$. This simplification is due to the experimental evidence that the main failure mode to be considered for RTDs is drift (Balaban et al., 2009). Overtime exposure to high temperatures can cause a drift in the measurements up to several degrees per year; shock and vibration generate strain in resistive wires of the RTDs and change their characteristics. In what follows, the quantitative analysis on the RTD-MSPM is done.

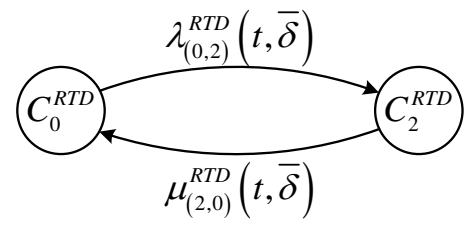

Fig. 4 Drift case of RTD-MSPM model 


\subsection{Estimation of the RTD-MSPM Parameters}

RTDs contain a sensing element whose resistance changes with fluid temperature. Well-type RTDs are assembled into thermowells containing the sensing elements, sheaths and insulation materials, whereas wet-type RTDs are designed for direct immersion into the fluid (Yun et al., 2012). Two types of RTDs are shown in Fig. 5 (Hashemian, 2011).

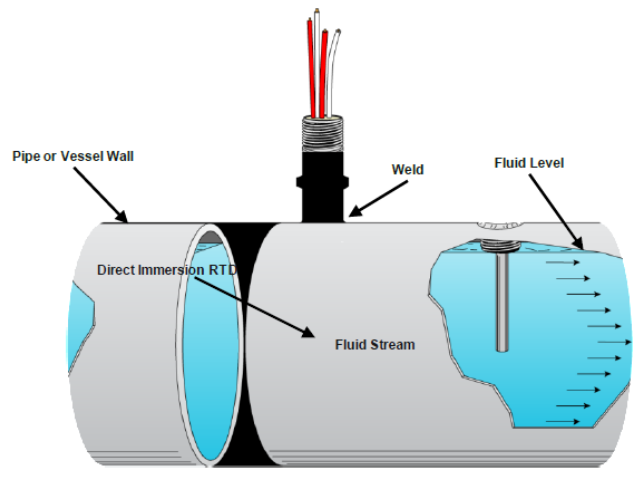

Wet-type RTD

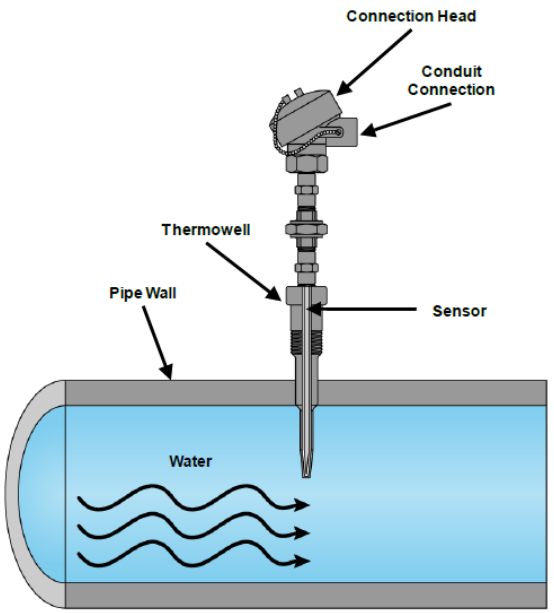

Well-type RTD

Fig. 5 Two types of RTDs (Hashemian, 2011)

The performance of RTDs is characterized by their measurement accuracy $\sigma$ and response time $\tau$. The former characterizes the RTD accuracy in measuring a sudden temperature change (Wei et al., 2013), whereas the latter is a pivotal indicator that measures how quickly the RTD responds to a sudden and significant temperature change (Yun et al., 2012). On one hand, being $\tau$ the time the RTD needs for reaching $63.2 \%$ of a sudden temperature change, $\tau$ is also representative of $\sigma$; on the other hand, since some failure modes might affect the fluid temperature and some others such as bias, freezing, loss of signal showing constant measured values even if the RTD is degraded and, thus, $\tau$ cannot be considered as performance metric, both $\tau$ and $\sigma$ are to be simultaneously considered for defining the RTD failure domain.

Under normal reactor operation conditions, effects of intrinsic properties (intrinsic shape and material properties, for example) are negligible on its own performance because they cannot be altered once the RTD is manufactured, such as $\tau$ and $\varepsilon$ (Hashemian, 1994). Instead, component aging $t$ and uncertainty of air gap size $\delta$ 
between the bottom of the thermowell and the RTD sensing tip due to contamination, metallurgical changes, moisture or dirt entering, mechanical shock, etc. are more likely to cause RTD drift (Hashemian, 2011; Swanson, 2007).

Aging can affect RTD performance with different degradation modes; for example, the sensing element resistance increases under tensile stress and decreases with compression stress that varies with time $t$, resulting in off-calibration, increase in $\tau$, reduced insulation resistance, erratic output, wiring problems, etc. Among these, sensor off-calibration and $\tau$ changes are the most relevant features to be monitored (Hashemian, 2011).

Moreover, we assume $\tau$ to heavily depend on air gap size $\delta$ between the RTD sensing tip and the bottom of the thermowell (Hashemian, 2013), even though also debris, dirt, and metal shavings entering the thermowell during installation, and/or moisture entering the insulation material during operation can, also cause an off-design of air gap that prevents the RTD from reaching the very bottom of its thermowell. Moreover, RTD movement in the thermowell due to vibration, thermal, or mechanical shock can cause the RTD sensing tip to displace away from the bottom of the thermowell (Hashemian, 2011) and calibration drift (Yun et al., 2012).

In conclusion, in what follows, $\tau$ is identified as the RTD drift physical variable $Y$ being degrading due to $t$ and $\delta$, as in Eq. (3):

$$
Y=\tau(t, \delta)
$$

Given a RTD failure threshold $\gamma_{Y}$ not to be exceeded by $\tau(t, \delta)$ during operation, then, its limit-state function can be formulated as:

$$
G=Y-\gamma_{Y}=\tau(t, \delta)-\gamma_{Y}
$$

To partition the RTD safety domain $S$ from its failure domain $F$, we firstly build a physical mathematical relationship between $\tau, t$ and $\delta$ based on the experimental data listed in Table 1 and 2 (Hashemian, 2011; Yun et al., 2012).

Table 1 Experimental data for $\tau$ at fixed $t$ and $\delta=0$ (Yun et al., 2012)

\begin{tabular}{|c|c|c|c|c|c|}
\hline Aging Time $t[\mathrm{yr}]$ & 0 & 2 & 4 & 5 & 6 \\
\hline Response Time $\tau[\mathrm{s}]$ & 2.1 & 4.4 & 4.8 & 5.0 & 5.2 \\
\hline Variance & 1.67 & 0.77 & 0.72 & 0.77 & 0.67 \\
\hline
\end{tabular}


Table 2 Experimental data for $\tau$ at $t=0$ and fixed $\delta$ (Hashemian, 2011; Yun et al., 2012)

\begin{tabular}{|c|c|c|c|c|c|c|c|c|c|c|c|}
\hline Air gap size $\delta[\mathrm{mm}]$ & 0 & 0 & 0.2 & 0.4 & 0.5 & 0.6 & 0.8 & 1.0 & 1.0 & 1.5 & 2.0 \\
\hline Response time $t[\mathrm{~s}]$ & 0.9 & 3.3 & 4.1 & 5.0 & 2.94 & 5.9 & 6.5 & 3.33 & 7.5 & 3.48 & 3.58 \\
\hline
\end{tabular}

Fig. 6 shows the trend of $\tau$ as long as $t$ increases, independently from the air gap contamination (i.e., $\delta=0$ ) when data in Table 1 are used as interpolation data. Fig. 7 shows the trend of $\tau$ as a function of $\delta$, when the RTD is new and data in Table 2 are used as interpolation data (i.e., aging $t=0$ ) (Yun et al., 2012).

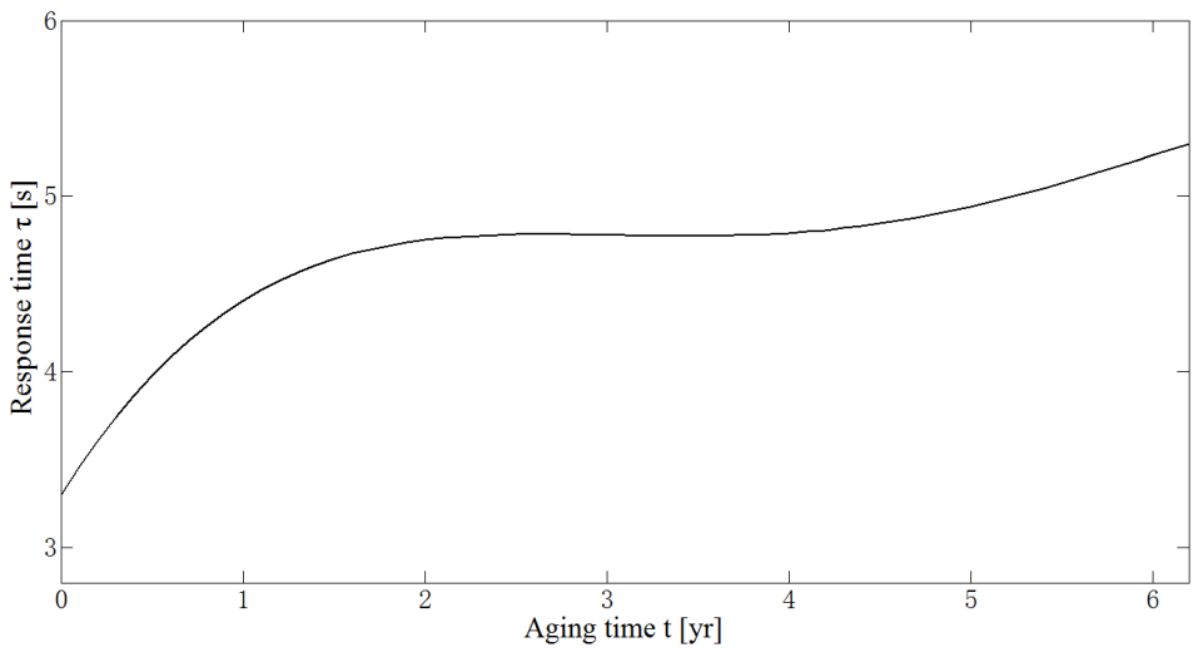

Fig. $6 \tau(t, 0)$

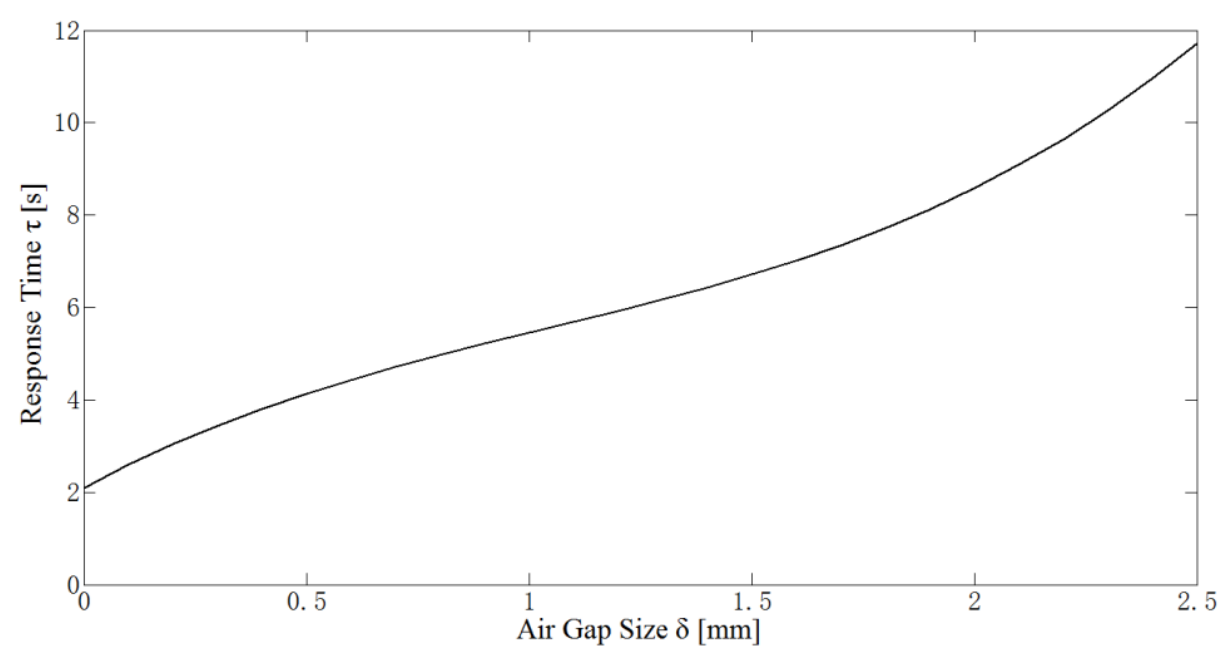

Fig. $7 \tau(0, \delta)$

The function $\tau(t, \delta)$ as it is plotted in Fig. 8 is obtained by resorting to Eq. (5):

$$
\tau(t, \delta)=\alpha_{t} \cdot \tau(t-1, \delta)
$$

where $\tau(0, \delta)$ is the curve of Fig. 7 and the factor $\alpha_{t}$ accounts for the changes of response time $\tau$ with the increase of $t$, by scaling the $\tau(t, 0)$ using the scale factor $\alpha_{t}$ : 


$$
\alpha_{t}=\frac{\tau(t)}{\tau(t-1)}
$$

where, $\alpha_{1}=\tau(1) / \tau(0)$. Table 3 reports the estimate of $\alpha_{t}$ for six discrete aging times $t$.

Table 3 Estimate of scale factors at six discrete aging times

\begin{tabular}{|c|c|c|c|c|c|c|c|}
\hline Aging time $t[\mathrm{yr}]$ & 0 & 1 & 2 & 3 & 4 & 5 & 6 \\
\hline Response time $\tau[\mathrm{s}]$ & 2.1040 & 3.5750 & 4.3818 & 4.7367 & 4.8525 & 4.9415 & 5.2163 \\
\hline Weighting factor $\alpha_{t}$ & $/$ & 1.70 & 1.23 & 1.08 & 1.02 & 1.02 & 1.06 \\
\hline
\end{tabular}

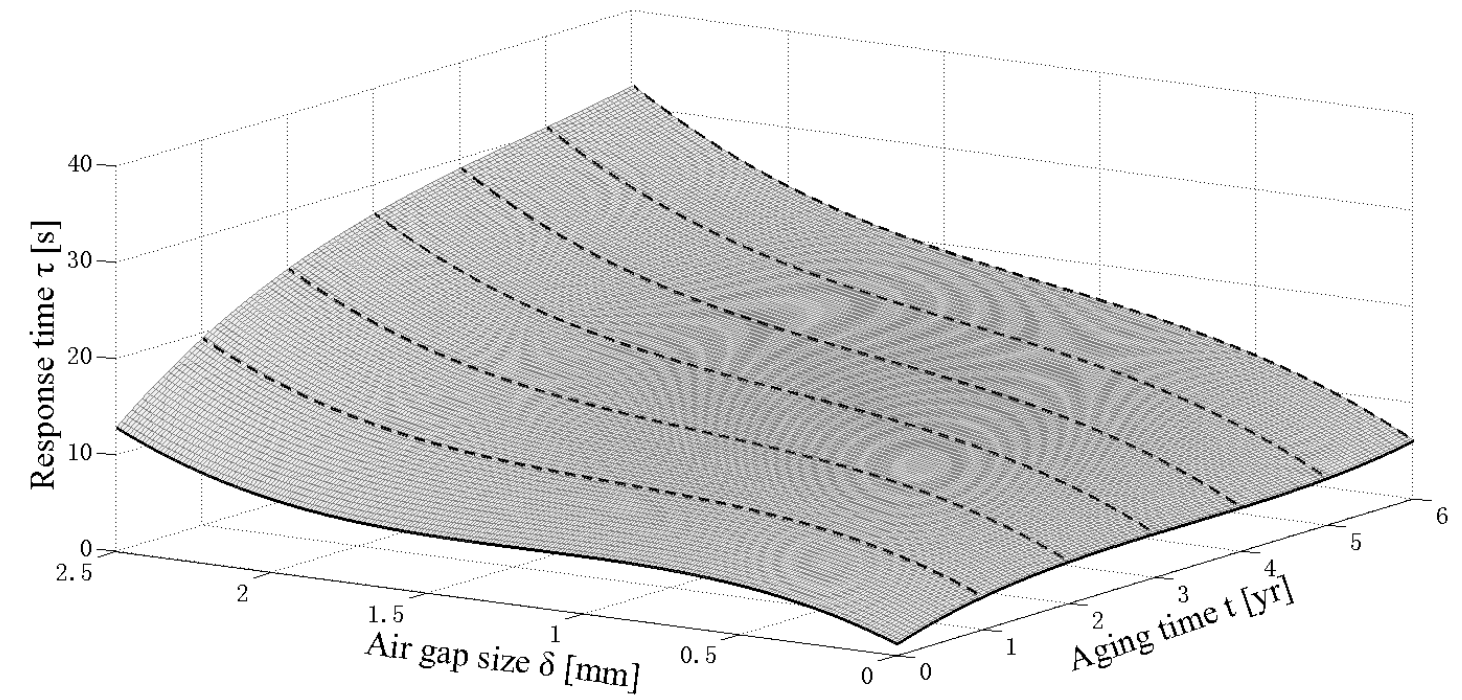

Fig. $8 \tau(t, \delta)$

As mentioned in (Hashemian, 2011), the $\tau$ of a well-type RTD usually ranges in [4s, 8s]; hence, the RTD failure threshold $\gamma_{\mathrm{Y}}$ is here set equal to $8 \mathrm{~s}$. The Cumulative Distribution Function (CDF) $P_{s}(t, \delta)$ of the RTD new-to-drift-failure mode that can account for the stochasticity of the process and of the uncertainties affecting the degradation (for example, the initial air gap size $\delta_{0}$ and the noise affecting the air gap size $\delta_{t}$ due to the vibration) can be found by running $N_{M}$ Monte Carlo simulations, as follows (see Table 4 for the list of parameters):

- For each trial, at the initial time $t=0$, we sample the value of $\delta_{0}$ from the uniform distribution $U(0,1)$ as initial air gap size.

- At each $t$ that increases with the time step $d t$, the value $d \delta_{t}$ is sampled from a normal distribution $N(0,0.025 t)$; thus, $\delta=\delta_{0}+d \delta_{t}$.

- At each $t$ within the mission time $\left[t_{0}, t_{m}\right], \tau$ is estimated using the curve $\tau(t, \delta)$ of Fig. 8. If the value of $\tau$ exceeds the threshold $\gamma_{Y}$, the RTD is assumed to 
fail at time $t$ with air gap size $\delta$.

Table 4 Setting of Parameters for Monte Carlo Simulation

\begin{tabular}{|c|c|c|c|c|}
\hline Parameter & Description & Unit & Value & Distribution \\
\hline$N_{M}$ & Simulation Times & $/$ & $10^{4}$ & $/$ \\
\hline$\gamma_{Y}$ & Response Time Threshold & $\mathrm{s}$ & 8 & $/$ \\
\hline$d t$ & Time interval & $\mathrm{yr}$ & $1 / 100$ & \\
\hline$t_{0}$ & Initial time & $\mathrm{yr}$ & 0 & $/$ \\
\hline$t_{m}$ & Mission time & $\mathrm{yr}$ & 6 & $\mathrm{U}(0,1)$ \\
\hline$\delta_{0}$ & Initial Air Gap Size & $\mathrm{mm}$ & $/$ & $\mathrm{N}(0,0.025 \mathrm{t})$ \\
\hline$d \delta_{t}$ & Noise of Air Gap Size & $\mathrm{mm}$ & $/$ & \\
\hline
\end{tabular}

Pictorially, we can show the evolution of $\tau(t, \delta)$ for each trial, as sketched in Fig. 9: for a sampled $\delta_{0}$ (equal to $0.12 \mathrm{~mm}$ ), the air gap size oscillates during the RTD life around $\delta_{0}$ (see in Fig. 9(a)); on the other hand, the response time $\tau$, stochastically changes with the increase of time $t$ (in Fig. 9(b)) and, thus, the transition between states $C_{0}^{R T D}$ and $C_{2}^{R T D}$ (see Fig. 4) of drift failure mode is determined when the response time $\tau$ reaches the failure threshold $\gamma_{Y}$, as shown in Fig. 9(c).

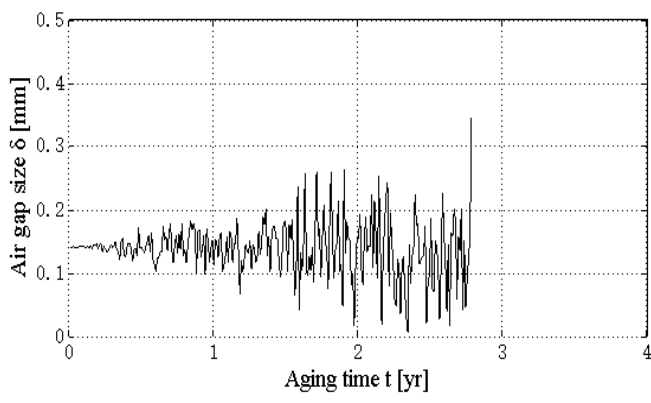

(a)

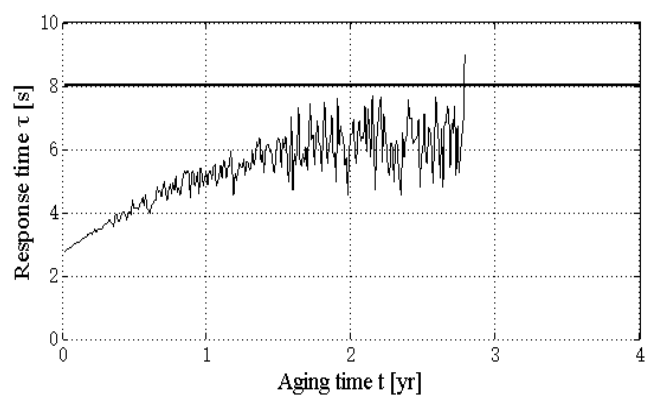

(b)

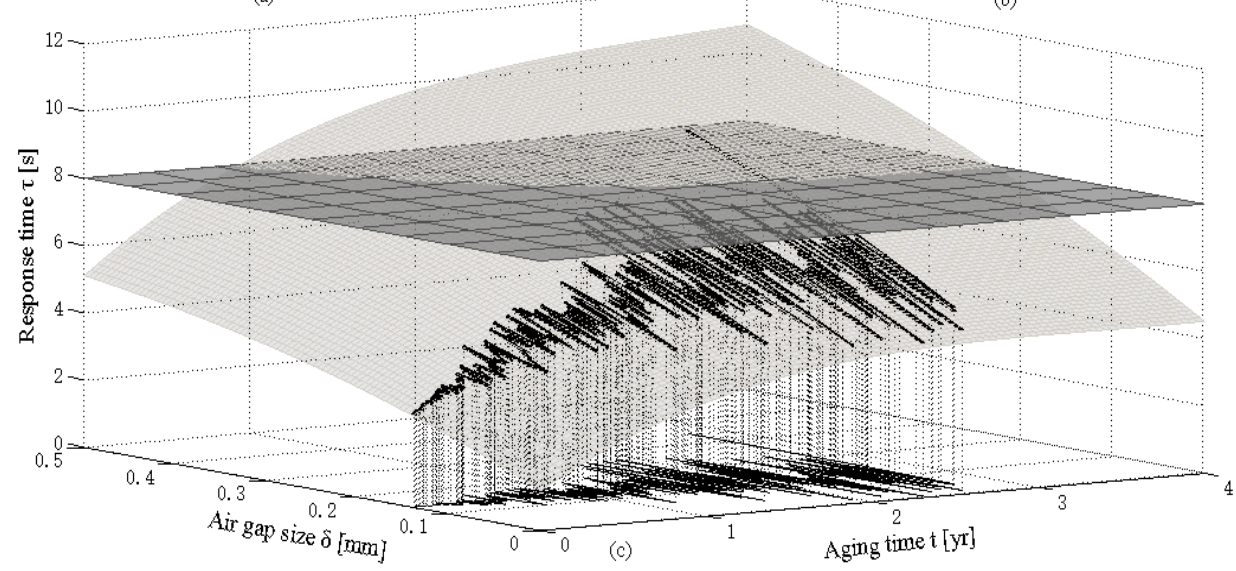

Fig. 9 One trial of MC simulation: (a) the stochastic path of air gap size $\delta$ changing with the aging $t$; (b) the evolution of response time $\tau$ with the aging $t$; (c) the simulated path response time $\tau$ with respect to $\delta$ and $t$ on the safety domain of the fitting curved surface 
After the $N_{M}$ trials of $\mathrm{MC}$ simulations have been run, the conditional Probability Density Function (PDF) $p_{S}(t \mid \delta)$ and conditional Cumulative Distribution Function (CDF) $P_{S}(t \mid \delta)$ of the RTD New-to-drift transition of Fig. 4 can be empirically built (shown in Fig. 10 and 11, respectively) and used to calculate the conditional failure rate $\lambda_{S}(t \mid \delta)$ :

$$
\begin{aligned}
& F_{S}(t<T<t+\Delta t \mid(T>t, \delta))=\frac{F_{S}(t<T<t+\Delta t \mid \delta)}{F_{S}(T>t \mid \delta)}=\frac{F_{S}(T<t+\Delta t \mid \delta)-F_{S}(T<t \mid \delta)}{1-F_{S}(T<t \mid \delta)}=\frac{P_{S}(t+\Delta t \mid \delta)-P_{S}(t \mid \delta)}{1-P_{S}(t \mid \delta)} \\
& \lambda_{S}(t \mid \delta)=\lim _{\Delta t \rightarrow 0} \frac{F_{S}(t<T<t+\Delta t \mid(T>t, \delta))}{\Delta t}=\lim _{\Delta t \rightarrow 0} \frac{P_{S}(t+\Delta t \mid \delta)-P_{S}(t \mid \delta)}{\Delta t} \cdot \frac{1}{1-P_{S}(t \mid \delta)}=\frac{p_{S}(t \mid \delta)}{R_{S}(t \mid \delta)}
\end{aligned}
$$

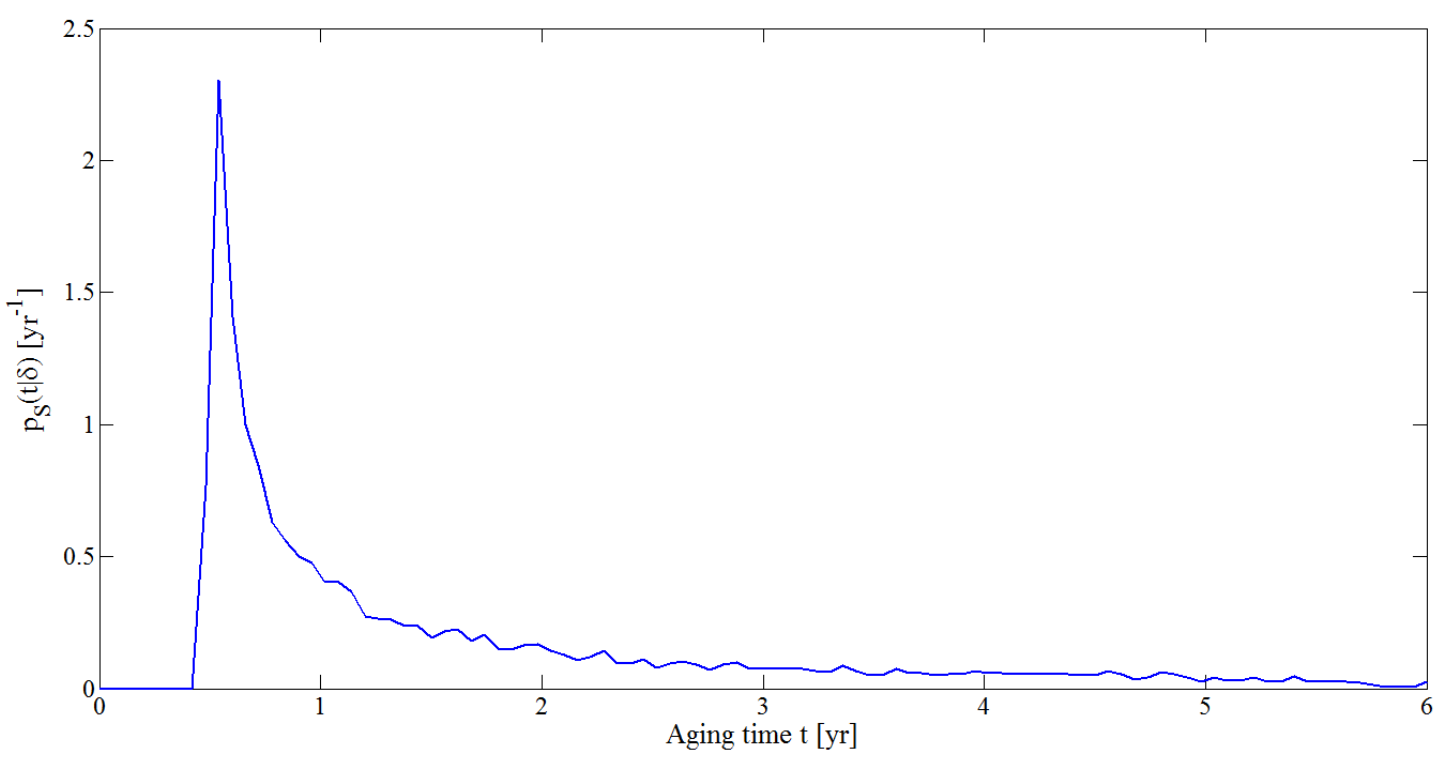

Fig. 10 Conditional probability density function of RTD New-to-drift failure mode

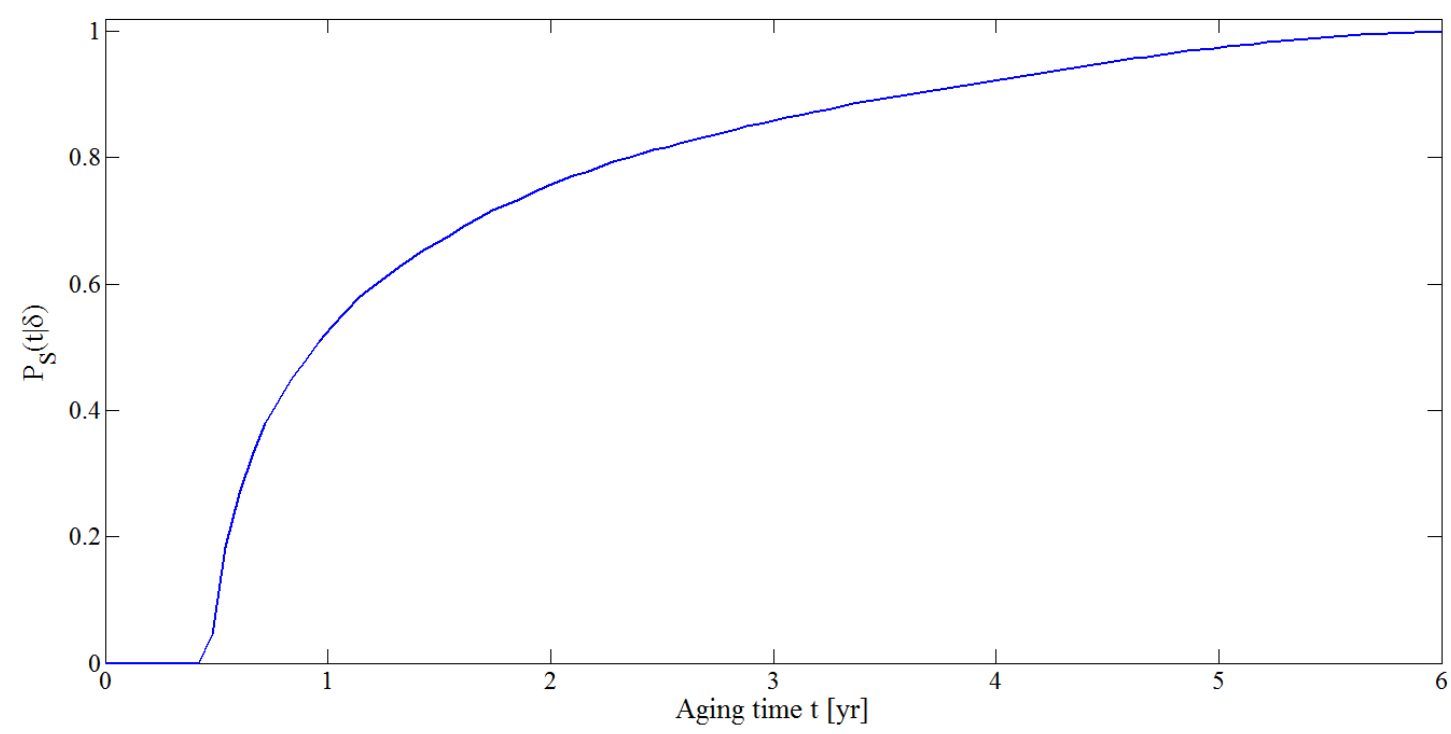

Fig. 11 Conditional cumulative distribution function of RTD New-to-drift failure mode 


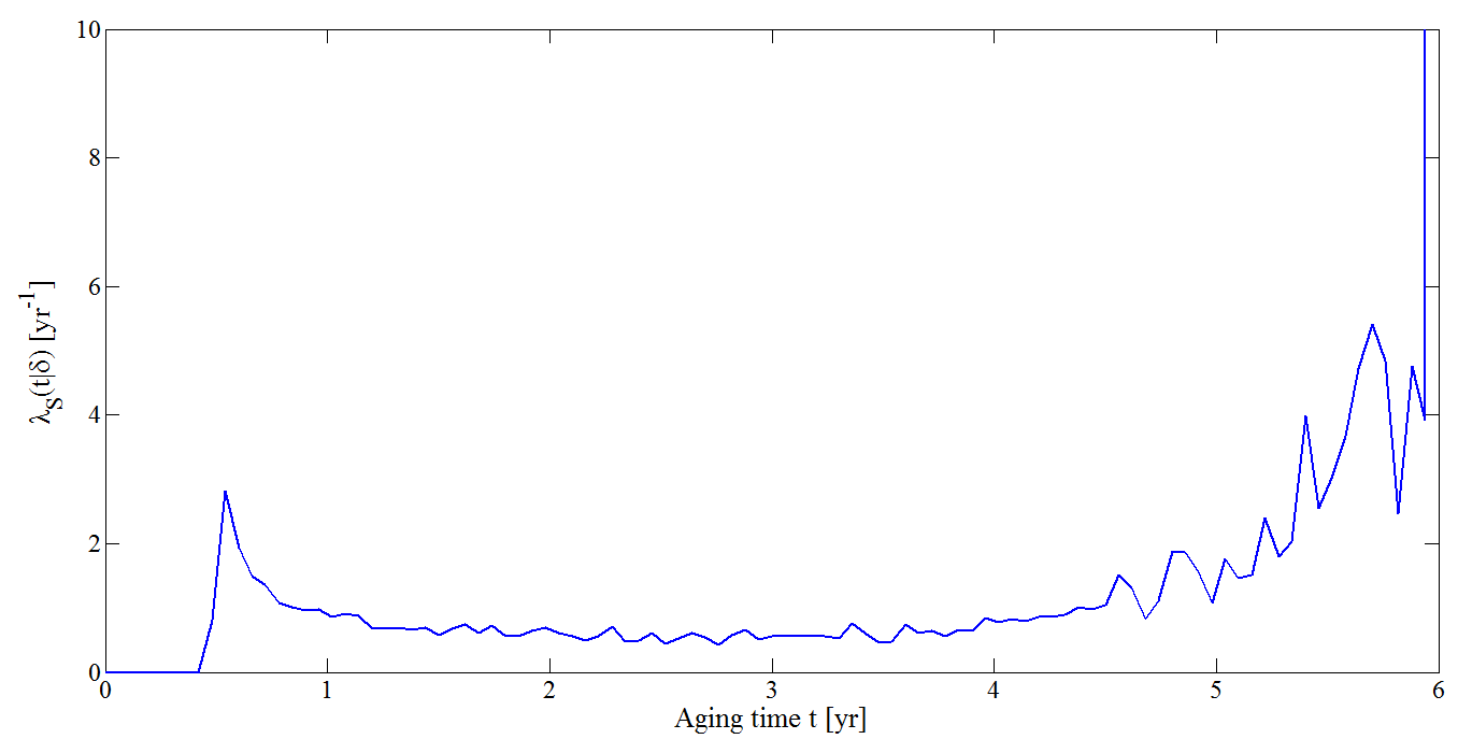

Fig. 12 Conditional failure rate of RTD New-to-drift failure mode

It is worth mentioning that the conditional CDF $P_{S}(t \mid \delta)$ of Fig. 11 describing the uncertain timing of RTD New-to-drift failure mode shows a sharp increase in $[0.5,1.5]$ yr, after which it starts to level off to reach $P_{S}(t \mid \delta)$ at 5.8yr. Therefore, the failure rate $\lambda_{S}(t \mid \delta)$ of Fig. 12 shows the typical infant mortality and wear out periods, and tends to be constant in the useful life, which coincides with a general bath-tub curve, but with non-constant values along life.

\subsection{Comparison with Markov Chain Model}

The results shown in Section 2.2 are compared with those of a MCM to describe a component failure in a binary-state modeling framework and neglecting any physical modeling of component degradation features. The equivalent of the RTD-MSPM of Fig. 4 is shown in the RTD-MCM of Fig. 13, where states " 0 " and "1" represent no-failure state and failure state respectively, and the transition rates between them $\lambda_{S}$ and $\mu_{S}$ are constant values ( $\lambda_{S}$ is equal to $1 \mathrm{E}-4 / \mathrm{hr}$ and $\mu_{S}$ to 0 (US: EPRI, 2008)). Therefore, the RTD unreliability (i.e., failure state probability) $P_{S}(t)$ can be calculated by means of MCM quantitative analysis and is plotted in Fig. 14.

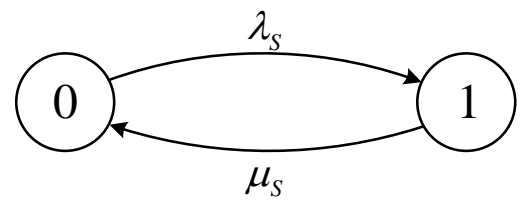

Fig. 13 Schematic diagram of RTD-MCM (without repair) 
Fig. 14 shows the results obtained from MSPM compared with those from MCM. The $P_{S}(t \mid \delta)$ (obtained by MSPM) is close to the $P_{S}(t)$ (obtained by MCM), which, however, overestimates the unreliability of the RTD. The degradation process modeling in the MSPM is more realistic than in MCM, and the latter is not able to guarantee the drift onset beyond a threshold value (especially at low aging).

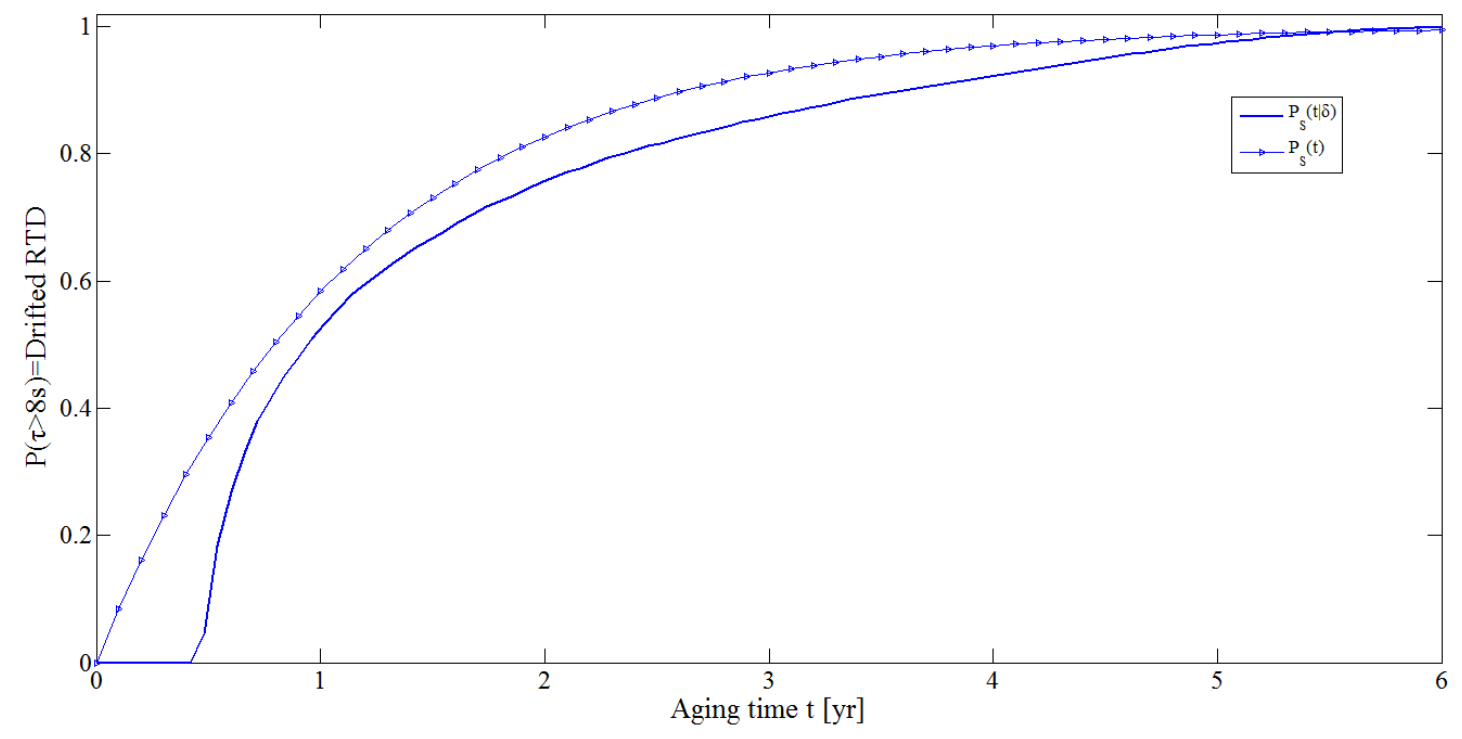

Fig. 14 Failure state probability obtained from MSPM and MCM approaches

\section{The System-Level MSPM for a Digital I\&C System}

In Section 2 we have shown how it is possible to resort to a MSPM framework to build a realistic model of a component/module that is embedded into a system. Now, the general framework of a MSPM approach is presented, when all the components of a Digital I\&C system are considered. As shown in Fig. 15, we propose a modular scheme that integrates the component-level and/or subsystem-level models into a system-level structure (Gulati and Dugan, 1997; Wang et al., 2015). Attention should be paid to the definition of system failure modes and to the identification of the component degradation states with their dependencies and the uncertainties, as well as on the inter-component dependencies by a qualitative and quantitative screening of the system behavior (i.e., by Failure Modes and Effects Analysis (FMEA) (Zio, 2007)). In this work, the components degradation process, their failure modes and the overall system behavior is modelled by implementing physics model at both component level or/and subsystem level, which consider inter-component and intra-components 
dependencies and uncertainties.

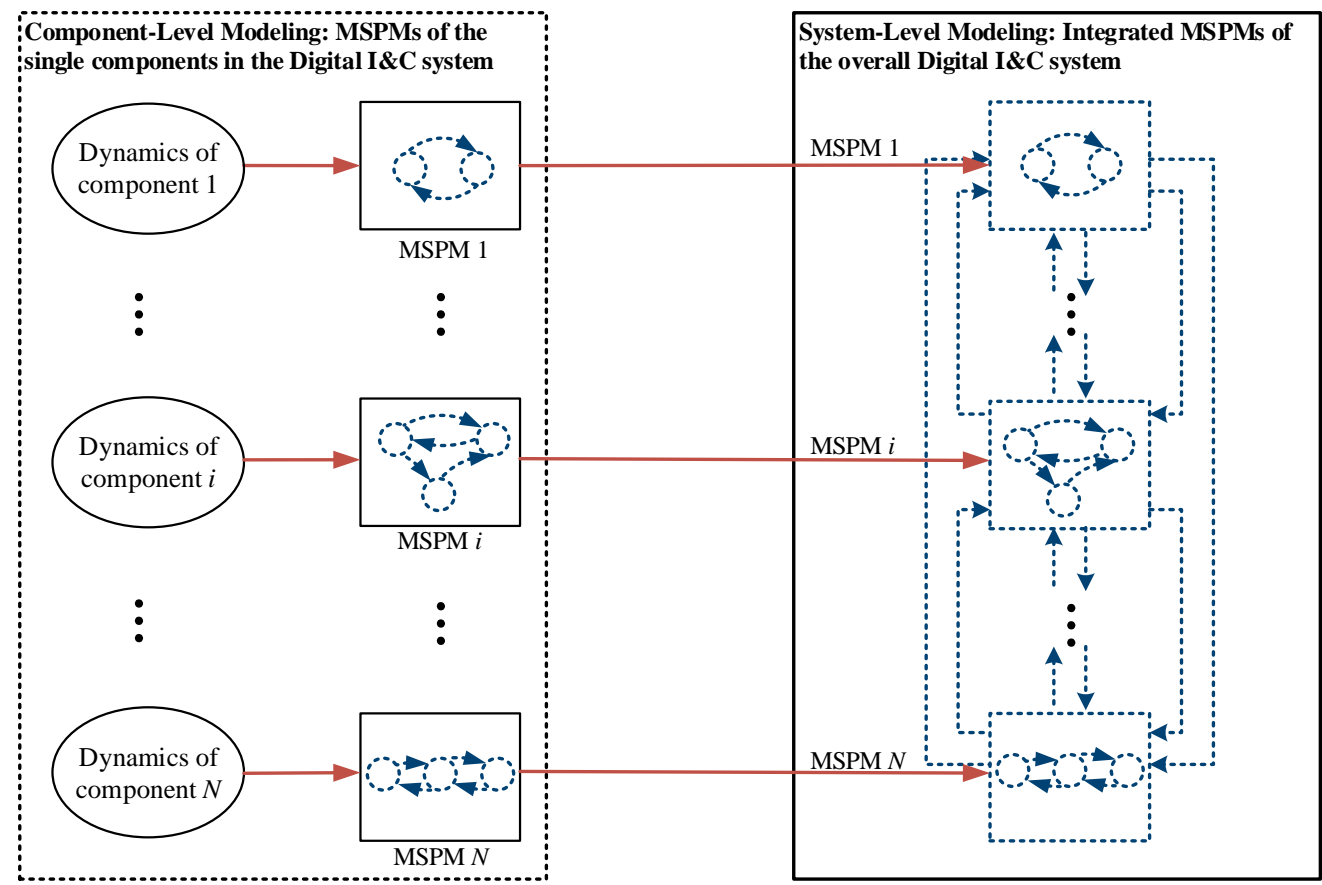

Fig. 15 General framework of a component-and system-level MSPM-based approach for a digital I\&C systems

With respect to the inter-component degradation physics, the following assumptions hold for each $i$-th component degradation MSPM. Provided that we are able to build, for each $i$-th component, its component-level MSPM, the system-level MSPM can be built, as shown in Fig. 16, where:

- Inter-components operating logic determines the layout of the $N+1$ layers, where layer " $L$ " " and layer " $L$ "N" are the "System functioning $\left(L^{0}\right)$ " and "System failure $\left(L^{N}\right)$ " states, respectively. Any intermediate layer " $L^{l ",}, l=$ $1, \cdots, N-1$ represents the module or submodule operating logic, where the component can be grouped in subsystems for similarity of task, location, characteristics, etc. (Inter-components (e.g., cascading failures) and intracomponents (e.g., components common cause failures) dependencies and uncertainties should be accounted for in detail for the sake of the model accuracy).

- $\quad L_{m}^{l}, m=1,2, \cdots, M^{l}$, is the system degradation state at progression level $m$, for the module failure mode $l$.

- All states $L_{m}^{l}$ are repairable states with repair rate $\mu_{L_{m}^{l} \rightarrow L^{0}}(t, \overline{\boldsymbol{\delta}})$ to $L^{0}$ 
(indicated by the dashed lines in Fig. 16) and failure rate $\lambda_{L_{m}^{l} \rightarrow L_{n}^{\omega}}(t, \overline{\boldsymbol{\delta}}), l, \omega=$ $1, \cdots, N-1, m=1,2, \cdots, M^{l}$, and $n=1,2, \cdots, M^{\omega}$ for the transition from state $L_{m}^{l}$ to any other state $L_{n}^{\omega}$ belonging to any layer $\omega \neq l$ (indicated by solid lines in Fig. 16).

- States $L_{m}^{l}$ lead to $L^{N}$ (i.e., system failure due to different failure modes) with failure rate $\lambda_{L_{m}^{l} \rightarrow L^{N}}(t, \overline{\boldsymbol{\delta}})$.

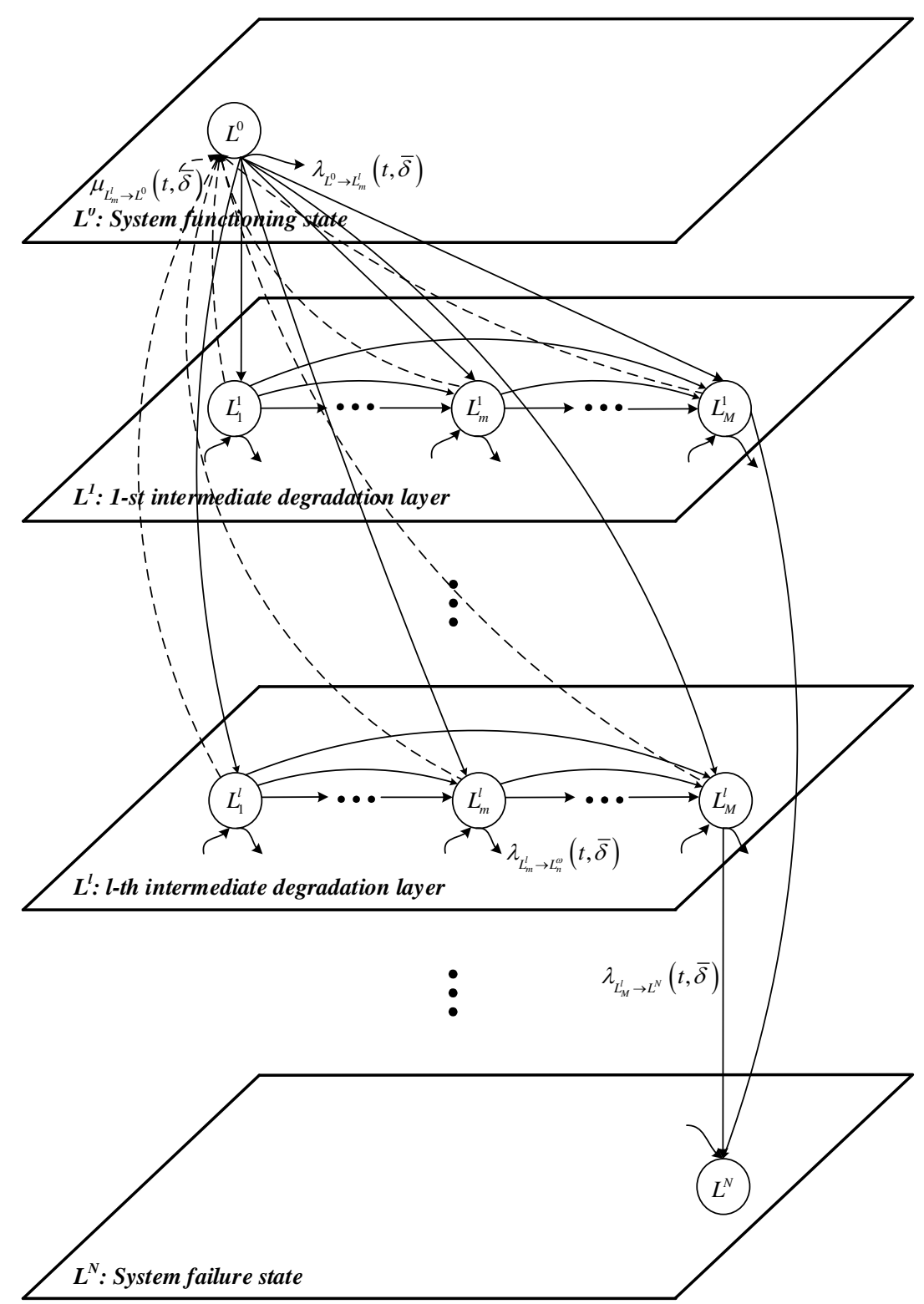

Fig. 16 The system-level MSPM degradation model

The construction of the system-level MSPM for the RPS of Fig. 1, thus, proceeds as follows: 
- Identification of system modules: BPL, LCL and RTB (see section 2).

- Definition of the $(N+1)=5$ layers.

The layers of the system-level MSPM of the RPS (see Fig. 17) are, therefore:

a) Layer $L^{0}$ is the "RPS functioning $\left(L^{0}\right)$ " state.

b) Layer $L^{1}$ models the degradation of the system due to either the submodule "S-A and BPL-A" or the submodule "S-B and BPL-B" failure, since the two submodules perform the same function in the system and the logic of such that the system goes into failure if at least one submodule fails. Due attention has to be, therefore, paid to the modeling the logic that process the PTSs. In layer $L^{1}$, states $L_{1}^{1}, \cdots, L_{m}^{1}, \cdots, L_{M}^{1}$ are states of the degradation of this module (shown in Fig. 17).

c) Layer $L^{2}$ models the degradation of the LCL module. Due to the operational logic of this module, the system goes into failure state only if both LCL-A and LCL-B fail, namely, the LCL module goes into state $L_{A N D}^{2}$ that is shown in Fig. 17. States $L_{1}^{2}, \cdots, L_{m}^{2}, \cdots, L_{M}^{2}$ are states of the degradation of LCL module.

d) Layer $L^{3}$ models the degradation of the RTB, from which the shutdown signal is directly sent to the power supply system and to the CRDM. States $L_{1}^{3}, \cdots, L_{m}^{3}, \cdots, L_{M}^{3}$ are the states of degradation of the RTB.

e) Layer $L^{4}$ is the "RPS failure $\left(L^{4}\right)$ " state.

- Identification of possible cascading failures affecting more than one layer/module.

- Identification of possible common cause failures among components belonging to different layers/modules.

- Quantification of the uncertainties in the probabilities of transition between states and layers/modules.

For completeness sake, in Fig. 17, $\mu_{L_{m}^{l} \rightarrow L^{0}}(t, \overline{\boldsymbol{\delta}})(l=1,2,3,4)$ is the repair transition rate from the state $m$ of the $l$-th layer to the "RPS functioning $\left(L^{0}\right)$ " state, as a function of time $t$ and of the factors $\overline{\boldsymbol{\delta}}$ affecting the degradation, $\lambda_{L^{0} \rightarrow L_{m}^{l}}(t, \overline{\boldsymbol{\delta}})$ is the 
failure transition rate from the "RPS functioning $\left(L^{0}\right)$ " state to the state $m$ of the $l$-th layer, $\lambda_{L_{m}^{l} \rightarrow L_{n}^{\omega}}(t, \overline{\boldsymbol{\delta}})(\omega=1,2,3,4)$ is the failure transition rate from the state $m$ of the $l$-th layer to the state $n$ of the $\omega$-th layer, and $\lambda_{L_{M}^{1} \rightarrow L^{4}}(t, \overline{\boldsymbol{\delta}}), \lambda_{L_{A N D}^{2} \rightarrow L^{4}}(t, \overline{\boldsymbol{\delta}})$, and $\lambda_{L_{M}^{3} \rightarrow L^{4}}(t, \overline{\boldsymbol{\delta}})$ are failure rates from the worst degradation states of each layer/module to the RPS failure state $L^{4}$.

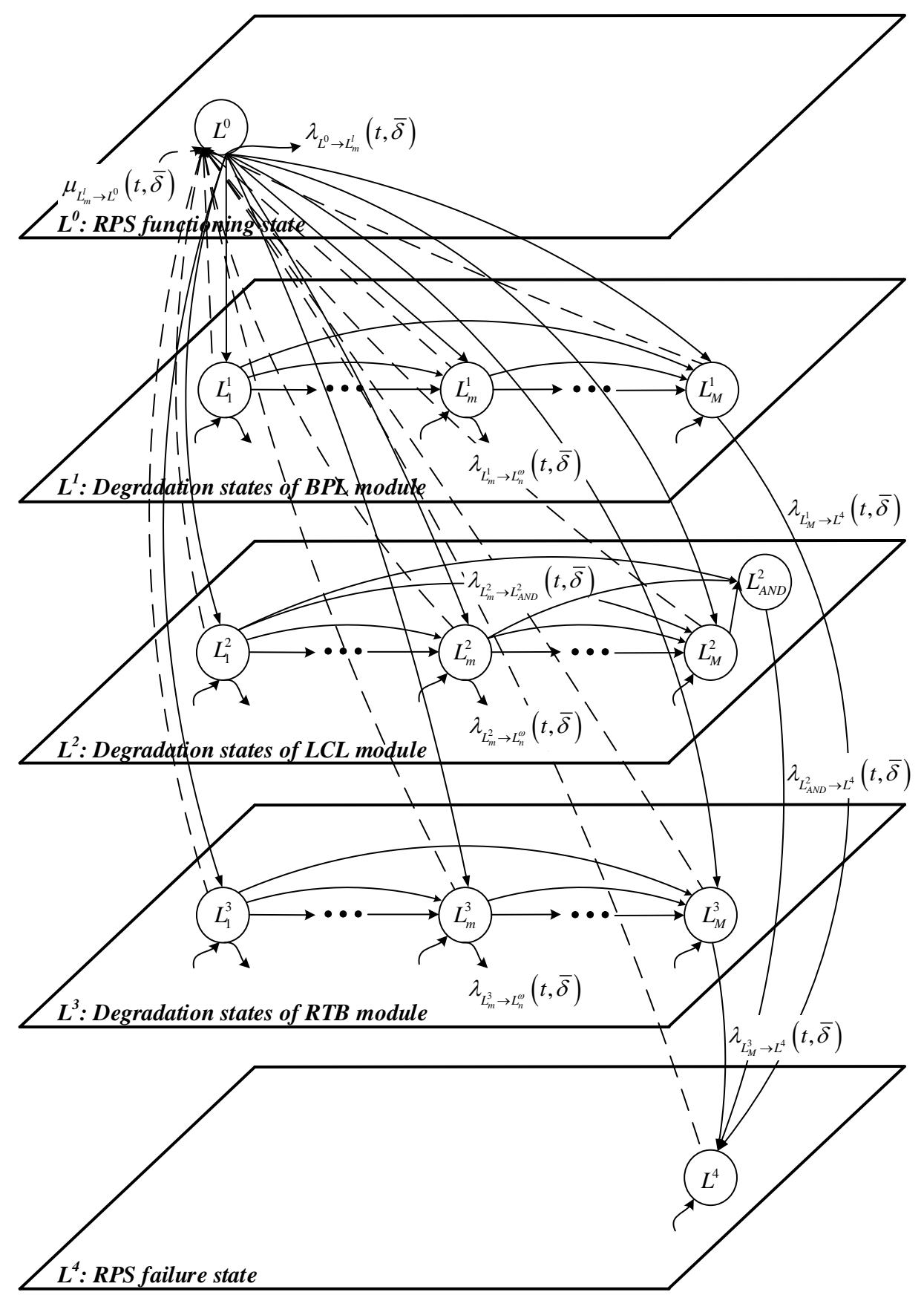

Fig. 17 The system-level degradation model of the RPS digital I\&C system

The objective of the system-level degradation modeling effort is the quantification 
of the state probability vector $\overline{\boldsymbol{P}}(t, \overline{\boldsymbol{\delta}})=\left\{p_{L^{0}}(t, \overline{\boldsymbol{\delta}}), p_{L_{1}^{1}}(t, \overline{\boldsymbol{\delta}}), \cdots, p_{L_{m}^{l}}(t, \overline{\boldsymbol{\delta}}), \cdots\right.$, $\left.p_{L_{M}^{4}}(t, \overline{\boldsymbol{\delta}}), p_{L^{5}}(t, \overline{\boldsymbol{\delta}})\right\}$, which can be obtained by Monte Carlo simulation to generate random walks across layers and within the MSPM describing each module, as illustrated in section 2. Without loss of generality, we will present a system-level MSPM where only the RTD-MSPM of section 2.1 is considered, whereas all the other components are assumed to obey a binary behavior (safe/failed). Therefore, the RPS system-level MSPM sketched in Fig. 19, whose states are described in Table 5, can be built:

- State $L^{0}$ is assumed as the system initial state, where all the components are new at $t=0$.

- State $L_{1}^{1}$ corresponds to the onset of the RTDs New-to-drift failure mode that may lead to system failure state $L^{4}$ when its response time exceeds the failure threshold (solid edges with transition rates represent the stochastic phenomena herein modeled).

- Absorbing states $L_{2}^{1}, L_{3}^{1}, L_{2}^{2}$ and $L_{A N D}^{2}$, deterministically lead without any stochasticity to the RPS system failure.

- Time-varying transition rates of the RTD sensors are taken from those of the RTD-MSPM of Section 2.2 (see Fig. 12), whereas the transition rates of binary-state components (i.e., BPLs, LCLs and RTB) are taken from public databases (BPL and LCL failure rates $\lambda_{B}$ and $\lambda_{L}$ are equal to $1 \mathrm{E}-6 / \mathrm{hr}$ and $5 \mathrm{E}-$ $6 / \mathrm{hr}$ respectively (US: EPRI, 2008), with the common cause factor $\beta=0.1$; whereas RTB failure rate $\lambda_{R}$ is equal to $4.3 \mathrm{E}-8 / \mathrm{hr}$ (IAEA, 1992)).

Table 5 Identification of states in RPS system-level MSPM

\begin{tabular}{|c|l|}
\hline State & \multicolumn{1}{|c|}{ Description } \\
\hline$L^{0}$ & RPS functioning state. \\
\hline$L_{1}^{1}$ & Drift onset in one or the other RTD sensor. \\
\hline$L_{2}^{1}$ & Either one or the other BPL fails to send out PTSs. \\
\hline$L_{3}^{1}$ & Common cause failure of BPL-A and BPL-B. \\
\hline$L_{1}^{2}$ & Either one or the other LCL fails to produce the ESS. \\
\hline$L_{2}^{2}$ & Common cause failure of LCL-A and LCL-B. \\
\hline$L_{A N D}^{2}$ & Both LCLs fail to produce the ESS. \\
\hline$L_{1}^{3}$ & RTB fails during operation. \\
\hline$L^{4}$ & RPS failure state. \\
\hline
\end{tabular}




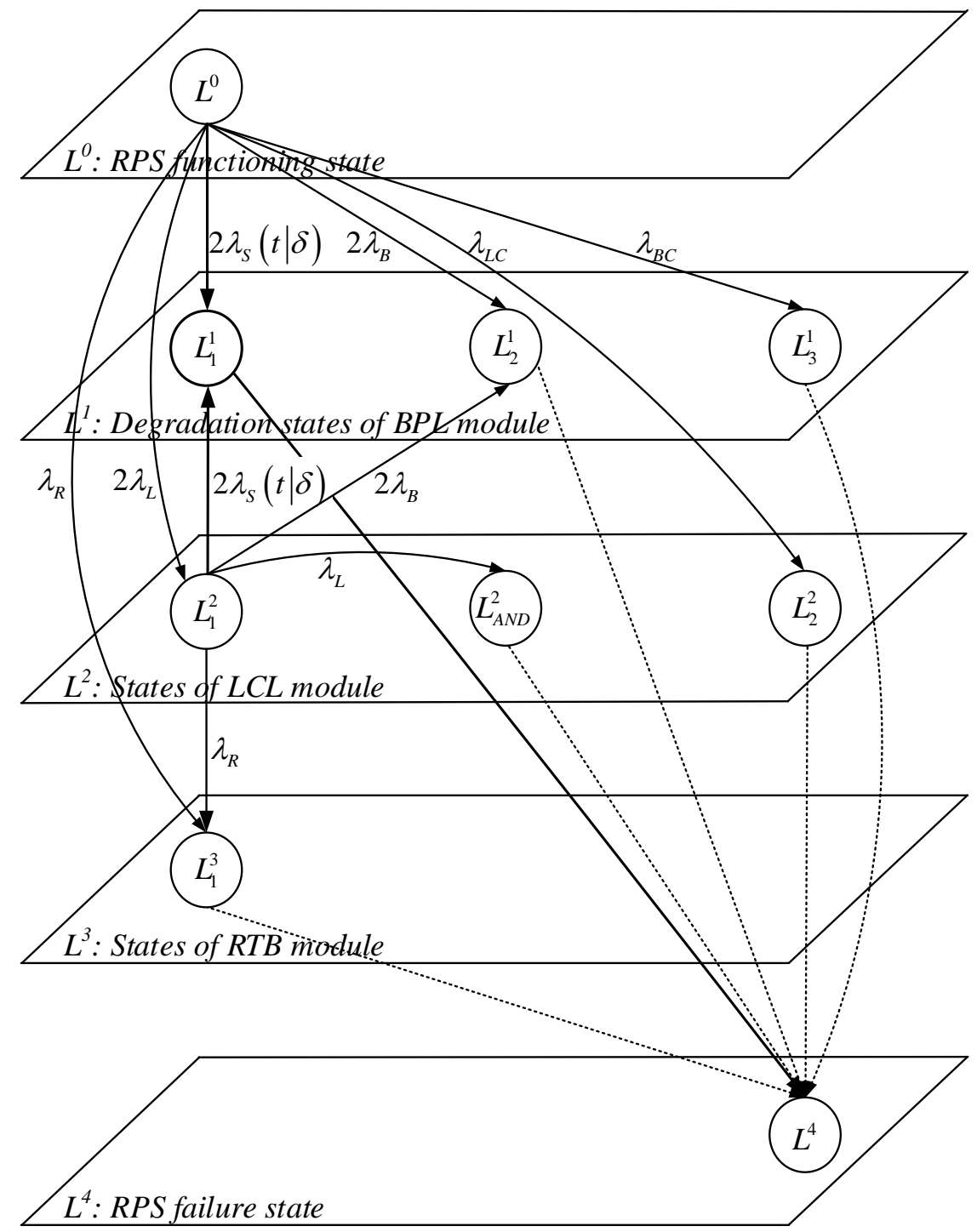

Fig. 19 The RPS system-level MSPM integrating RTD New-to-drift failure mode

After $N_{M}$ trials of the Monte Carlo simulation, whose flowchart is shown in Fig. 20 (where the inner shadowed loop corresponds to the Monte Carlo simulation adopted in Section 2.2 for the estimation of the RTD drift onset time), the calculated RPS unreliability $P(t \mid \delta)$ is plotted in Fig. 21, and compared with the system unreliability $P(t)$ obtained by solving a binary-states homogeneous continuous-time discrete-state Markov Chain Model (i.e., the RPS-MCM), where also the RTDs are assumed to have a constant transition rate equal to $1 \mathrm{E}-4 / \mathrm{hr}$ (US: EPRI, 2008). It can be seen that the RTD-MCM overestimates the system unreliability, which makes the design over conservative, or the maintenance over demanding, especially at the early stage of the 
system life.

Sample the BPLs, LCLs and RTB transition times (from safe to failed, or vice versa)

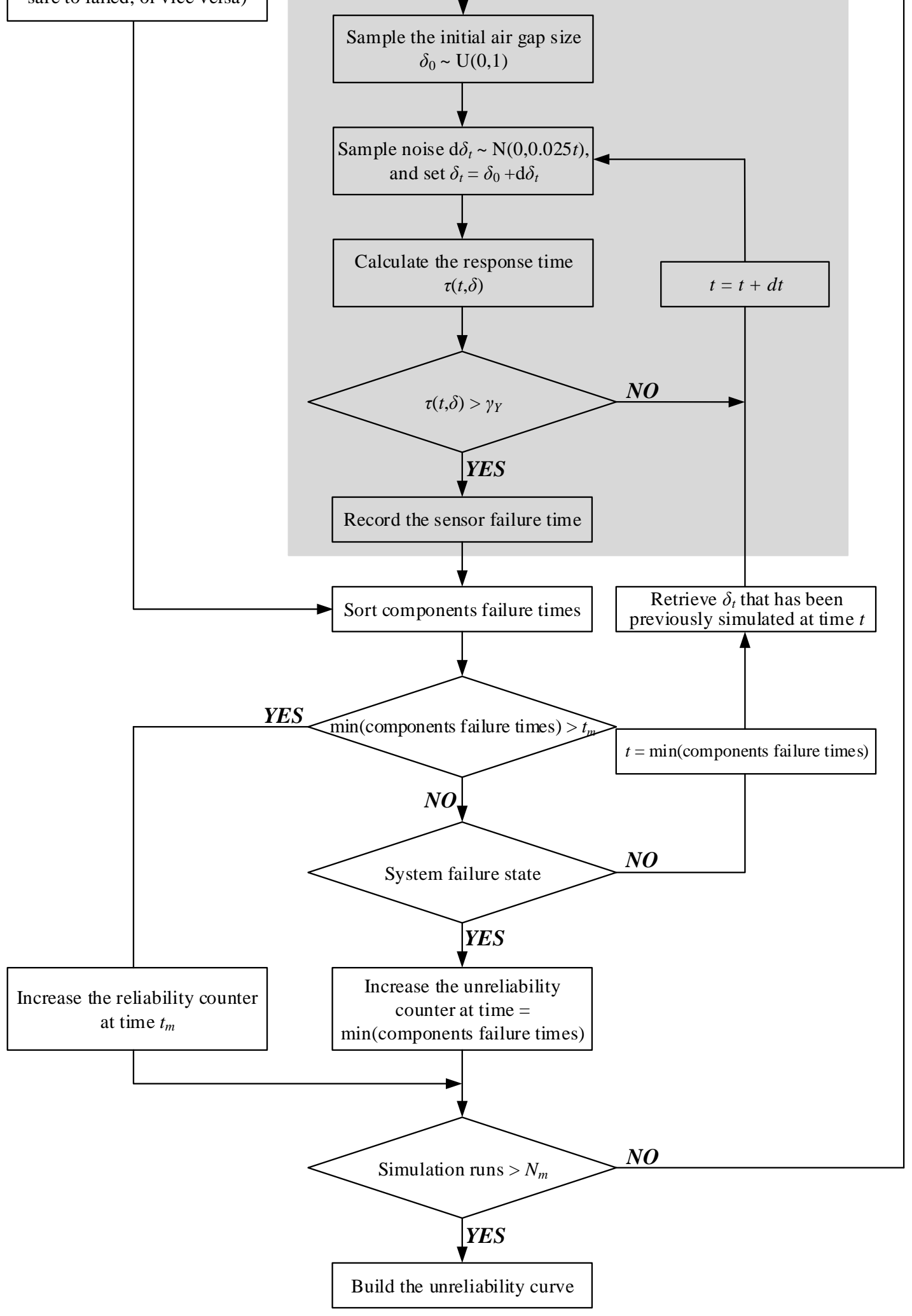

Fig. 20 The flowchart of the Monte Carlo Simulation for the system-level MSPM 


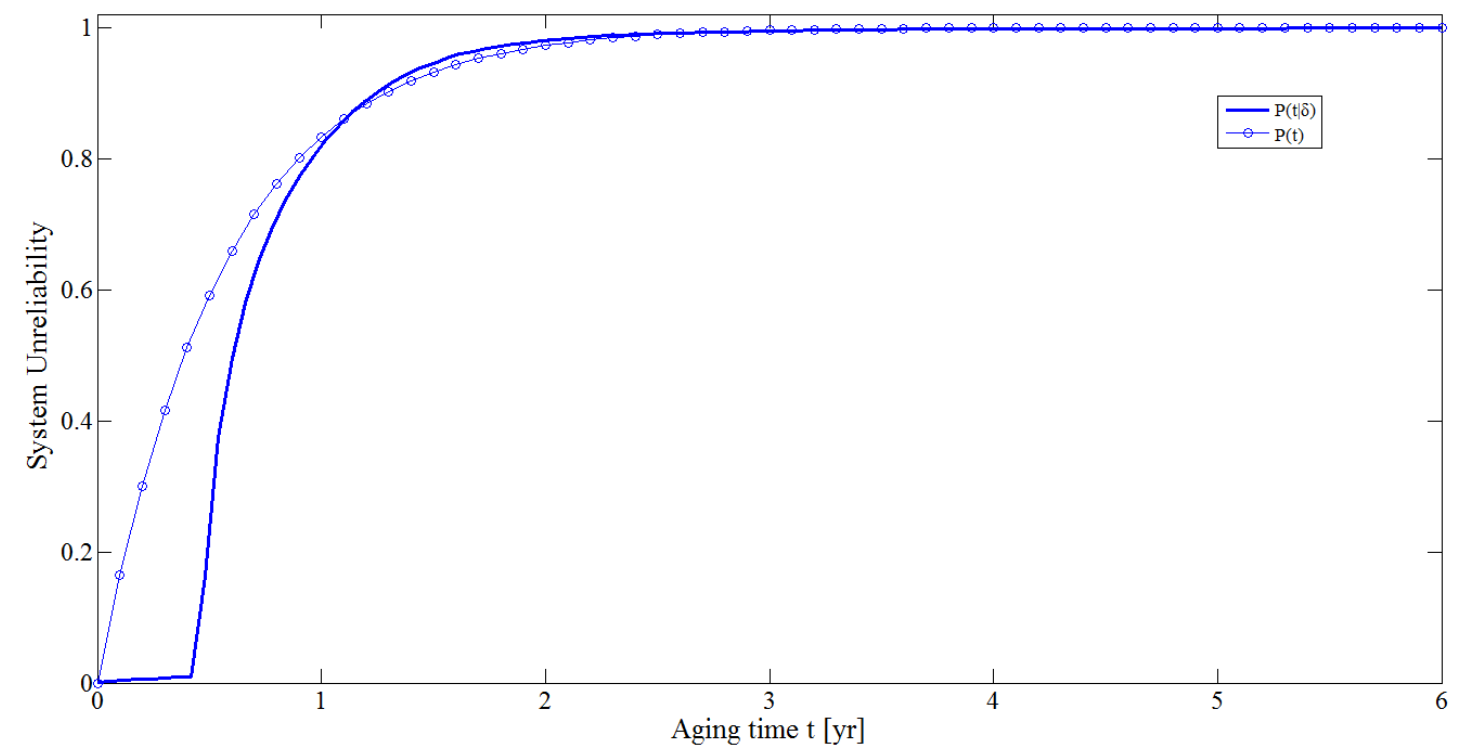

Fig. 21 System unreliability obtained from RPS-MSPM and RPS-MCM framework

\section{Conclusions}

A MSPMs framework has been proposed for reliability modeling and assessment of digital I\&C systems in NPPs. The designers and operators efforts needed to gather the necessary, and quite detailed, physics of failure data that are to be treated within the MSPM framework makes the method primarily applicable to critical NPPs components. Indeed, in this work a RTD was considered to develop the component-level MSPM model, being a typical signal input source of digital I\&C systems that critically affects its reliability. Monte Carlo simulation has been used for estimating the degradation state probability (i.e. drift-failure state probability) in the RTD-MSPM, by sampling the stochastic evolution of the air gap size, under deterministic aging. The comparison of MCM and MSPM results shows that with realistic assumptions and available knowledge, MSPM provides a better and more complete representation of the component degradation progression.

The modular modeling approach proposed has been applied to develop a systemlevel MSPM model taking for a typical Reactor Protection System(RPS) as benchmark.

The results of the illustrative application demonstrate that the proposed MSPM approach can well explain environmental conditions, aging and degradation of failure events, besides timing and sequencing which can also be solved by traditional dynamic methods (e.g., MCM). 


\section{Reference}

Aldemir, T., Guarro, S., Kirschenbaum, J., Mandelli, D., Mangan, L.A., Bucci, P., Yau, M., Johnson, B., Elks, C., Ekici, E., Stovsky, M.P., Miller, D.W., Sun, X., Arndt, S.A., Nguyen, Q., Dion J., 2009. A benchmark implementation of two dynamic methodologies for the reliability modeling of digital instrumentation and control systems. US Nuclear Regulatory Commission, Office of Nuclear Regulatory Research.

Aldemir, T., Miller, D. W., Stovsky, M. P., Kirschenbaurr, J., Bucci, P., Fentiman, A.W., Mangan, L.T., 2006. Current State of Reliability Modeling Methodologies for Digital Systems and Their Acceptance Criteria for Nuclear Power Plant Assessments. US Nuclear Regulatory Commission.

Aldemir, T., Stovsky, M. P., Kirschenbaum, J., Mandelli, D., Bucci P., Mangan, L.A., Miller, D.W., Sun, X., Ekici, E., Guarro, S., Yau, M., Johnson, B., Elks, C., Arndt. S.A., 2007. Dynamic reliability modeling of digital instrumentation and control systems for nuclear reactor probabilistic risk assessments. US Nuclear Regulatory Commission, Office of Nuclear Regulatory Research.

Balaban, E., Saxena, A., Bansal, P., Goebel, K. F., Curran, S., 2009. "Modeling, detection, and disambiguation of sensor faults for aerospace applications." Sensors Journal, IEEE, No. 9(12), pp. 1907-1917.

Baraldi, P., Di Maio, F., Genini, D., Zio, E., 2015. "Comparison of data-driven reconstruction methods for fault detection.” IEEE Transactions in reliability, Vol. 64, No. 3, pp. 852-860.

Boskovic, J. D., Mehra, R. K., 2002. "Stable adaptive multiple model-based control design for accommodation of sensor failures." In Proceedings of the American Control Conference, pp. 2046-2051.

Boudali, H., Dugan, J. B., 2006. "A continuous-time Bayesian network reliability modeling, and analysis framework." Reliability, IEEE Transactions on 55, No. 1, pp. 86-97.

Broy, P., Chraibi, H., Donat, R., 2011. "Using Dynamic Bayesian Networks to solve a 
dynamic reliability problem." Advances in Safety, Reliability and Risk Management (ESREL 2011), 55.

Bucci, P., Mangan, L. A., Kirschenbaum, J., Mandelli, D., Aldemir, T., Arndt, S. A., 2006. "Incorporation of Markov reliability models for digital instrumentation and control systems into existing PRAs." Proceedings of NPIC\&HMIT.

Castello, C., Sanyal, J., Rossiter, J., Hensley, Z., New, J., 2014. "Sensor Data Management, Validation, Correction, and Provenance for Building Technologies." In Proceedings of the ASHRAE Annual Conference and ASHRAE Transactions.

Dehlinger, J., Dugan, J. B., 2008. "Analyzing dynamic fault trees derived from modelbased system architectures." Nuclear Engineering and Technology 40, No. 5, pp. $365-374$.

Di Maio, F., Colli, D., Zio, E., Liu, T., Tong, J. J., 2015. "A Multi-State Physics Modeling approach for the reliability assessment of Nuclear Power Plants piping systems." Annals of Nuclear Energy 80, pp. 151-165.

Di Maio, F., Secchi, P., Vantini, S., Zio, E., 2011. "Fuzzy C-Means Clustering of Signal Functional Principal Components for Post-Processing Dynamic Scenarios of a Nuclear Power Plant Digital Instrumentation and Control System.” IEEE Transactions on Reliability, Volume 60, No. 2, pp. 415-425.

Ferdinand, P., Magne, S., Laffont, G., 2013. "Optical fiber sensors to improve the safety of nuclear power plants.” In Asia Pacific Optical Sensors Conference 2013, pp. 89242G-89242G.

Fernandeza, L., Marcoa, S., Gutierrez-Galvez, A., 2015. "Robustness to sensor damage of a highly redundant gas sensor array." Sensors and Actuators B: Chemical, Vol. 218, pp. 296-302.

Fleming, K. N., Unwin, S. D., Kelly, D., Lowry, P. P., Toloczko, M. B., Layton, R. F., Youngblood, R., Collins, D., Huzurbazar, A. V., Williams, B., Heasler, P. G., 2010. "Treatment of Passive Component Reliability in Risk-Informed Safety Margin Characterization." Idaho National Laboratory, INL/EXT-10-20013, Idaho Falls, Idaho 2010, pp. 1-210. 
García-Martín, J., Gómez-Gil, J., Vázquez-Sánchez, E., 2011. "Non-destructive techniques based on eddy current testing." Sensors 11, No. 3, pp. 2525-2565.

Garvey, D. R., Hines, J. W., 2006. "An adaptive distance measure for use with nonparametric models." Conference: NPIC and HMIT 2006, Albuquerque, US.

Guarro, S., Yau, M., Dixon, S., 2012. "Applications of the Dynamic Flowgraph Methodology to Dynamic Modeling and Analysis," Proceedings of the 11th International Conference on Probabilistic Safety Assessment and Management (PSAM 11), Helsinki, Finland, June 25-29.

Gulati, R., Dugan, J. B., 1997. "A modular approach for analyzing static and dynamic fault trees." In Reliability and Maintainability Symposium. 1997 Proceedings, Annual, pp. 57-63, IEEE.

Hashemian, H. M., 1994. “Aging Characteristics of Nuclear Plant RTDs and Pressure Transmitters." The 4th International Topical Meeting on Nuclear Thermal Hydraulics, Operations and Safety, Taipei, Taiwan, April 6-8.

Hashemian, H. M., 2011. Measurement of Dynamic Temperatures and Pressures in Nuclear Power Plants. Ph.D. Thesis.

Hashemian, H. M., 2013. "Response time testing of temperature sensors using loop current step response method.” International Journal of Nuclear Energy Science and Technology 7, No. 3, pp. 209-230.

IAEA, 1992. Case study on the use of PSA methods: Assessment of technical specifications for the reactor protection system instrumentation. IAEATECDOC-669.

Kaiser, K. A., Gebraeel, N. Z., 2009. "Predictive maintenance management using sensor-based degradation models." Systems, Man and Cybernetics, Part A: Systems and Humans, IEEE Transactions on 39, No. 4, pp. 840-849.

Kim, H. J., Kim, J., 2014. "Reliability Analysis of Core Protection Calculator System using Petri Net." Probabilistic Safety Assessment and Management PSAM 12, Honolulu, Hawaii.

Lee, D. Y., Choi, J. G., Joon, L., 2006. "A safety assessment methodology for a digital 
reactor protection system." International Journal of Control Automation and Systems 4, No. 1, pp. 105-112.

Li, Y. F., Zio, E., Lin, Y. H., 2012. "A multistate physics model of component degradation based on stochastic Petri nets and simulation." Reliability, IEEE Transactions on 61, No. 4, pp. 921-931.

Lin, Y. H., Li, Y. F., Zio, E., 2015. "Integrating Random Shocks Into Multi-State Physics Models of Degradation Processes for Component Reliability Assessment." Reliability, IEEE Transactions on 64, No. 1, pp. 154-166.

Lisnianski, A., Levitin, G., 2003. Multi-State System Reliability: Assessment, Optimization and Applications. Singapore: World Scientific, Vol. 6.

Montalvo, C., García-Berrocal, A., Bermejo, J. A., Queralc, C., 2014. “Advanced surveillance of resistance temperature detectors in nuclear power plants." Annals of Nuclear Energy 65, pp. 35-40.

Rocco, C. M., Zio, E., 2013. "Global sensitivity analysis in a Multi-state Physics Model of Component Degradation based on a hybrid State-Space Enrichment and Polynomial Chaos Expansion approach." Reliability, IEEE Transactions on 62, No. 4, pp. 781-788.

Swanson, C., 2007. "Optimal temperature sensor selection: Achieving accurate temperature measurement." Euro-Asia Semiconductor, Vol. 29, No. 7, pp. 23-28.

Uchanin, V., Najda, V., 2011. “The development of eddy current technique for WWER steam generators inspection.” INTECH Open Access Publisher.

Uren, K. R., Van Schoor, G., Carel, P., Du Randb, C. P., Botha, A., 2015. “An integrated approach to sensor FDI and signal reconstruction in HTGRs-Part II: Case studies." Annals of Nuclear Energy, Vol. 87, Part 2, pp. 739-749.

Unwin, S. D., Lowry, P. P., Layton, R. F., Heasler, P. G., Toloczko, M. B., 2011. “Multistate physics models of aging passive components in probabilistic risk assessment." in Proc. Int. Topical Meeting Probabilistic Safety Assessment and Anal (PSA 2011), Wilmington, NC, USA, Mar. 13-17, Vol. 1, pp. 161-172.

Unwin, S. D., Lowry, P. P., Toyooka, M. Y., 2012. "Reliability Models of Aging Passive 
Components Informed by Materials Degradation Metrics to Support Long-Term Reactor Operations." Nuclear Science and Engineering, No. 171.1, pp. 69-77.

US: EPRI, 2008. Utility requirement document annex a reliability data base for passive ALWR PRAs.

Wang, W., Zhao, J., Tong, J. J., Zhou, J. X., Xiao, P., 2015. "Evaluation Method of Reliability Indicator of Reactor Protection System.” Atomic Energy Science and Technology, Vol. 49 (6), pp. 1101-1108.

Wei, J. F., Sun, L. Q., Zhang, K., 2013. "Study on the influence of the response characteristics of a temperature sensor on the measurement accuracy of a waterabsorption-based high-energy laser energy meter." Measurement Science and Technology, Vol. 24055103.

Yun, D., Yacout, A. M., Vilim, R. B., 2012. "Modeling the Aging Effects of Nuclear Power Plant Resistance Temperature Detectors." 8th International Topical Meeting on Nuclear Plant Instrumentation, Control, and Human-Machine Interface Technologies 2012, NPIC and HMIT 2012: Enabling the Future of Nuclear Energy.

Zio, E., 2007. An Introduction to the Basics of Reliability and Risk Analysis. World Scientific Publishing.

Zio, E., Di Maio, F., 2009. "Processing Dynamic Scenarios from a Reliability Analysis of a Nuclear Power Plant Digital Instrumentation and Control System.” Annals of Nuclear Energy, Volume 36(9), pp. 1386-1399.

Zhou, S. L., Jiang, R. T., Liu, Y. Y., 2014. "Reliability assessment of nuclear power plant digital instrumentation and control system I: Challenges and research status of reliability assessment methodology." In Control and Decision Conference (2014 CCDC), pp. 1773-1778, IEEE. 